\title{
High-pressure vibrational and optical study of $\mathrm{Bi}_{2} \mathrm{Te}_{3}$
}

\author{
R. Vilaplana, ${ }^{1,}{ }^{*}$ O. Gomis, ${ }^{1}$ F. J. Manjón, ${ }^{2}$ A. Segura, ${ }^{3}$ E. Pérez-González, ${ }^{4}$ P. Rodríguez-Hernández, ${ }^{4}$ A. Muñoz, ${ }^{4}$ \\ J. González, ${ }^{5,6}$ V. Marín-Borrás, ${ }^{3}$ V. Muñoz-Sanjosé, ${ }^{3}$ C. Drasar, ${ }^{7}$ and V. Kucek ${ }^{7}$ \\ ${ }^{1}$ Centro de Tecnologías Físicas, MALTA Consolider Team, Universitat Politècnica de València, 46022 Valencia, Spain \\ ${ }^{2}$ Instituto de Diseño para la Fabricación y Producción Automatizada, MALTA Consolider Team, Universitat Politècnica de València, \\ 46022 Valencia, Spain \\ ${ }^{3}$ Instituto de Ciencia de Materiales de la Universidad de Valencia-MALTA Consolider Team-Departamento de Física Aplicada, Universitat \\ de València, 46100 Burjassot, Valencia, Spain \\ ${ }^{4}$ MALTA Consolider Team-Departamento de Física Fundamental II and Instituto Universitario de Materiales y Nanotecnología, \\ Universidad de La Laguna, La Laguna, Tenerife, Spain \\ ${ }^{5}$ DCITIMAC, MALTA Consolider Team, Universidad de Cantabria, Avda. de Los Castros s/n, 39005 Santander, Spain \\ ${ }^{6}$ Centro de Estudios de Semiconductores, Universidad de los Andes, Mérida 5201, Venezuela \\ ${ }^{7}$ Faculty of Chemical Technology, University of Pardubice, Studentská 95, 53210-Pardubice, Czech Republic
}

(Received 8 June 2011; revised manuscript received 25 July 2011; published 9 September 2011)

\begin{abstract}
We report an experimental and theoretical lattice dynamics study of bismuth telluride $\left(\mathrm{Bi}_{2} \mathrm{Te}_{3}\right)$ up to 23 GPa together with an experimental and theoretical study of the optical absorption and reflection up to $10 \mathrm{GPa}$. The indirect bandgap of the low-pressure rhombohedral $(R-3 m)$ phase $\left(\alpha-\mathrm{Bi}_{2} \mathrm{Te}_{3}\right)$ was observed to decrease with pressure at a rate of $-6 \mathrm{meV} / \mathrm{GPa}$. In regard to lattice dynamics, Raman-active modes of $\alpha-\mathrm{Bi}_{2} \mathrm{Te}_{3}$ were observed up to $7.4 \mathrm{GPa}$. The pressure dependence of their frequency and width provides evidence of the presence of an electronic-topological transition around $4.0 \mathrm{GPa}$. Above $7.4 \mathrm{GPa}$ a phase transition is detected to the $C 2 / \mathrm{m}$ structure. On further increasing pressure two additional phase transitions, attributed to the $C 2 / c$ and disordered bcc $(I m-3 m)$ phases, have been observed near 15.5 and 21.6 GPa in good agreement with the structures recently observed by means of $\mathrm{x}$-ray diffraction at high pressures in $\mathrm{Bi}_{2} \mathrm{Te}_{3}$. After release of pressure the sample reverts back to the original rhombohedral phase after considerable hysteresis. Raman- and IR-mode symmetries, frequencies, and pressure coefficients in the different phases are reported and discussed.
\end{abstract}

DOI: 10.1103/PhysRevB.84.104112

PACS number(s): 61.50.Ks, 62.50.-p, 78.20.Ci, 78.30.-j

\section{INTRODUCTION}

Bismuth telluride $\left(\mathrm{Bi}_{2} \mathrm{Te}_{3}\right)$ is a layered chalcogenide with a tremendous impact for thermoelectric applications. ${ }^{1}$ The thermoelectric properties of $\mathrm{Bi}_{2} \mathrm{Te}_{3}$ and their alloys have been extensively studied due to their promising operation in the temperature range of $300-400 \mathrm{~K}$. In fact $\mathrm{Bi}_{2} \mathrm{Te}_{3}$ is the material with the best thermoelectric performance at ambient temperature. ${ }^{2,3}$ Recently, it has been shown that $\mathrm{Bi}_{2} \mathrm{Te}_{3}$ can be exfoliated like graphene and that a single layer exhibits high electrical conductivity and low thermal conductivity so that a new nanostructure route can be envisaged to improve dramatically the thermoelectrical properties of this compound by means of either charge-carrier confinement or acousticphonon confinement. ${ }^{4,5}$

$\mathrm{Bi}_{2} \mathrm{Te}_{3}$ is a narrow bandgap semiconductor with tetradymite crystal structure $[R-3 m$, space group (S.G.) $166, Z=3] .^{6}$ This rhombohedral-layered structure is formed by layers, which contain five hexagonal close-packed atomic sublayers (Te-Bi-Te-Bi-Te) and is named a quintuple linked by van der Waals forces. The same layered structure is common to other narrow bandgap semiconductor chalcogenides, like $\mathrm{Bi}_{2} \mathrm{Se}_{3}$ and $\mathrm{Sb}_{2} \mathrm{Te}_{3}$, and has been found in $\mathrm{As}_{2} \mathrm{Te}_{3}$ at high pressures. ${ }^{7}$

$\mathrm{Bi}_{2} \mathrm{Te}_{3}$, as well as $\mathrm{Bi}_{2} \mathrm{Se}_{3}$ and $\mathrm{Sb}_{2} \mathrm{Te}_{3}$, has been recently predicted to behave as a topological insulator ${ }^{8}$; i.e., a new class of materials that behave as insulators in the bulk but conduct electrical current in the surface. The topological insulators are characterized by the presence of a strong spin-orbit (SO) coupling that leads to the opening of a narrow bandgap and causes certain topological invariants in the bulk to differ from their values in vacuum. The sudden change of invariants at the interface results in metallic, time-reversal invariantsurface states whose properties are useful for applications in spintronics and quantum computation. ${ }^{9,10}$ Therefore, in recent years a number of papers have been devoted to the search of the 3D-topological insulators among $\mathrm{Sb}_{2} \mathrm{Te}_{3}, \mathrm{Bi}_{2} \mathrm{Te}_{3}$, and $\mathrm{Bi}_{2} \mathrm{Se}_{3}$, and different works observed the features of the topological nature of the band structure in the three compounds. ${ }^{11-13}$

High-pressure studies are very useful to understand materials properties and design new materials because the increase in pressure allows us to reduce the interatomic distances and to finely tune the materials properties. It has been verified that the thermoelectric properties of semiconductor chalcogenides improve with increasing pressure, and that the study of the properties of these materials could help in the design of better thermoelectric materials by substituting external pressure by chemical pressure. ${ }^{14-18}$ Therefore, the electrical and thermoelectric properties of $\mathrm{Sb}_{2} \mathrm{Te}_{3}, \mathrm{Bi}_{2} \mathrm{Te}_{3}$, and $\mathrm{Bi}_{2} \mathrm{Se}_{3}$, as well as their electronic-band structure, have been studied at high pressures. ${ }^{19-27}$ In fact a decrease of the bandgap energy with increasing pressure was found in $\mathrm{Bi}_{2} \mathrm{Te}_{3} .{ }^{19,20}$ Furthermore, recent high-pressure studies in these compounds have shown a pressure-induced superconductivity ${ }^{28,29}$ that has further stimulated high-pressure studies. ${ }^{30}$ However, the pressure dependence of many properties of these layered chalcogenides is still not known. In particular the determination of the crystalline structures of these materials at high pressures has been a long puzzle ${ }^{15,23,31,32}$ and the space groups of the high-pressure phases of $\mathrm{Bi}_{2} \mathrm{Te}_{3}$ have been elucidated 
only recently by powder $\mathrm{x}$-ray diffraction measurements at synchrotron-radiation sources ${ }^{33,34}$ specially with the use of particle-swarm optimization algorithms for crystal-structure prediction. ${ }^{34}$

Recent high-pressure powder x-ray diffraction measurements have evidenced a pressure-induced electronic topological transition (ETT) in $\mathrm{Bi}_{2} \mathrm{Te}_{3}$ around $3.2 \mathrm{GPa}$ as a change in compressibility. ${ }^{29,31,32,35,36}$ An ETT or Lifshitz transition occurs when an extreme of the electronic-band structure, which is associated to a Van Hove singularity in the density of states, crosses the Fermi-energy level. ${ }^{37}$ This crossing, which can be driven by pressure, temperature, doping, etc., results in a change in the topology of the Fermi surface that changes the electronic density of states near the Fermi energy. An ETT is a 2.5 transition in the Ehrenfest description of the phase transitions so no discontinuity of the volume (first derivative of the Gibbs free energy) but a change in the compressibility (second derivative of the Gibbs free energy) is expected in the vicinity of the ETT. Anomalies in the phonon spectrum are also expected for materials undergoing an ETT ${ }^{38,39}$ and have been observed in a number of materials $\mathrm{s}^{40,41}$ as well as in $\mathrm{Sb}_{1.5} \mathrm{Bi}_{0.5} \mathrm{Te}_{3} .{ }^{31}$

The lattice dynamics of $\mathrm{Bi}_{2} \mathrm{Te}_{3}$ have been studied experimentally at room pressure ${ }^{42-44}$ and a recent study suggests that Raman spectroscopy can be used to monitor the number of single quintuple layers in nanostructured $\mathrm{Bi}_{2} \mathrm{Te}_{3}$, as in graphene. ${ }^{45}$ Theoretical studies of the lattice dynamics of $\mathrm{Bi}_{2} \mathrm{Te}_{3}$ at room pressure have also been performed; ${ }^{46-49}$ however, Raman measurements at high pressures have only been reported up to $0.5 \mathrm{GPa},{ }^{50}$ and to our knowledge there is no theoretical study of the lattice dynamics properties of $\mathrm{Bi}_{2} \mathrm{Te}_{3}$ under high pressure. As a part of our systematic study of the structural stability and the vibrational properties of the semiconductor chalcogenide family, we report in this work room-temperature Raman-scattering measurements in $\mathrm{Bi}_{2} \mathrm{Te}_{3}$ up to $23 \mathrm{GPa}$ together with total-energy and lattice-dynamical $a b$ initio calculations at different pressures. We discuss the recent observation of a pressure-induced ETT in the rhombohedral phase of $\alpha-\mathrm{Bi}_{2} \mathrm{Te}_{3}$ and study whether the Raman-scattering signal of the $\mathrm{Bi}_{2} \mathrm{Te}_{3}$ at pressures above 7.4 GPa match with the proposed high-pressure phases recently reported for this compound ${ }^{33,34}$ and which have also been found in $\mathrm{Sb}_{2} \mathrm{Te}_{3}$ at high pressures. ${ }^{51}$

\section{EXPERIMENTAL DETAILS}

We have used single crystals of $p$-type $\mathrm{Bi}_{2} \mathrm{Te}_{3}$ that were grown using a modified Bridgman technique. A polycrystalline ingot was synthesized by the reaction of stoichiometric quantities of the constituting elements $(5 \mathrm{~N})$. Afterward, the polycrystalline material was annealed and submitted to the growth process in a vertical Bridgman furnace. Preliminary room-temperature measurements on single crystalline samples $(15 \mathrm{~mm} \times 4 \mathrm{~mm} \times 0.3 \mathrm{~mm})$ yield in-plane electrical resistivity $\rho_{\perp \mathrm{c}}=1.7 \cdot 10^{-5} \Omega \mathrm{m}$ and Hall coefficient $\mathrm{R}_{\mathrm{H}}(\mathrm{B} \| \mathrm{c})=$ g $0.52 \mathrm{~cm}^{3} \mathrm{C}^{-1}$. Following the calculation presented in Ref. 52, the latter gives hole concentration of $7.2 \cdot 10^{18} \mathrm{~cm}^{-3}$ and minority electron concentration of $2.1 \cdot 10^{17} \mathrm{~cm}^{-3}$.

A small flake of the single crystal $(100 \mu \mathrm{m} \times$ $100 \mu \mathrm{m} \times 5 \mu \mathrm{m})$ was inserted in a membrane-type diamond anvil cell (DAC) with a 4:1 methanol-ethanol mixture as pressure-transmitting medium, which ensures hydrostatic conditions up to $10 \mathrm{GPa}$ and quasihydrostatic conditions between 10 and $23 \mathrm{GPa}^{53,54}$ Pressure was determined by the ruby luminescence method. ${ }^{55}$

Unpolarized room-temperature Raman-scattering measurements at high pressures were performed in backscattering geometry using two setups: (i) A Horiba Jobin Yvon LabRAM HR microspectrometer equipped with a TE-cooled multichannel CCD detector and a spectral resolution below $2 \mathrm{~cm}^{-1}$. HeNe laser (6328 ̊̊ line) was used for excitation. (ii) A Horiba Jobin Yvon T64000 triple-axis spectrometer with resolution of $1 \mathrm{~cm}^{-1}$. In this case an $\mathrm{Ar}^{+}$laser (6470 $\AA$ line) was used for excitation. In order not to burn the sample, power levels below $2 \mathrm{~mW}$ were used inside the DAC. This power is higher than that used in Raman measurements at room pressure due to superior cooling of the sample in direct contact with the pressure-transmitting media and the diamonds.

Optical transmission and reflection measurements under pressure were performed by putting the DAC in a home-built Fourier Transform infrared (FTIR) setup operating in the mid-IR region $\left(400-4000 \mathrm{~cm}^{-1}\right)$. The pressure-transmitting medium was $\mathrm{KBr}$. The setup consists of a commercial TEO400 FTIR interferometer by ScienceTech S.L., which includes a Globar thermal-infrared source and a Michelson interferometer, and a liquid-nitrogen cooled Mercury-Cadmium-Telluride (MCT) detector with wavelength cutoff at $25 \mu \mathrm{m}\left(400 \mathrm{~cm}^{-1}\right)$ from IR Associates Inc. A gold-coated parabolic mirror focuses the collimated IR beam onto a calibrated iris of 1 to $3 \mathrm{~mm}$ diameter. A gold-coated X15 Cassegrain microscope objective focuses the IR beam inside the DAC to a size of 70$200 \mu \mathrm{m}$. A second Cassegrain microscope objective collects the transmitted IR beam and sends it to the detector after being focused by another parabolic mirror. In the reflection configuration, a flat gold mirror is placed at $45^{\circ}$ before the focusing Cassegrain objective, blocking half of the IR beam. The half-beam let into the DAC is reflected by the sample, then by the flat gold mirror, and finally focused on the MCT detector by another parabolic mirror.

\section{AB INITIO CALCULATIONS}

Two recent works have reported the structures of the high-pressure phases of $\mathrm{Bi}_{2} \mathrm{Te}_{3}$ up to $52 \mathrm{GPa}^{33,34}$ The rhombohedral $(R-3 m)$ structure $\left(\alpha-\mathrm{Bi}_{2} \mathrm{Te}_{3}\right)$ is suggested to transform to the $C 2 / m\left(\beta-\mathrm{Bi}_{2} \mathrm{Te}_{3}, \mathrm{~S} . \mathrm{G} .12, Z=4\right)$ and the $C 2 / c$ $\left(\gamma-\mathrm{Bi}_{2} \mathrm{Te}_{3}\right.$, S.G. $\left.15, Z=4\right)$ structures above 8.2 and $13.4 \mathrm{GPa}$, respectively. ${ }^{34}$ Furthermore, a fourth phase $\left(\delta-\mathrm{Bi}_{2} \mathrm{Te}_{3}\right)$ has been found above $14.5 \mathrm{GPa}$ and assigned to a disordered bcc structure (Im-3m, S.G. 229, $Z=1) .^{33,34}$ In order to explore the relative stability of these phases in $\mathrm{Bi}_{2} \mathrm{Te}_{3}$ we have performed ab initio total-energy calculations within the density functional theory (DFT) ${ }^{56}$ using the plane-wave method and the pseudopotential theory with the Vienna ab initio simulation package (VASP) $)^{57}$ We have used the projector-augmented wave scheme $(\mathrm{PAW})^{58}$ implemented in this package. Basis set, including plane waves up to an energy cutoff of $320 \mathrm{eV}$, were used in order to achieve highly converged results and accurate descriptions of the electronic properties. We have used the generalized gradient approximation (GGA) 
for the description of the exchange-correlation energy with the $\mathrm{PBEsol}^{59}$ exchange-correlation prescription. Dense special $\mathrm{k}$-points sampling for the Brillouin zone (BZ) integration were performed in order to obtain very well-converged energies and forces. At each selected volume, the structures were fully relaxed to their equilibrium configuration through the calculation of the forces on atoms and the stress tensor. In the relaxed equilibrium configuration, the forces on the atoms are less than $0.002 \mathrm{eV} / \AA$ and the deviation of the stress tensor from a diagonal hydrostatic form is less than $1 \mathrm{kbar}(0.1 \mathrm{GPa})$. Since the calculation of the disordered bcc phase was not possible to do, we have attempted to perform calculations for the bcc-like monoclinic $C 2 / m$ structure proposed in Ref. 34. The application of DFT-based total-energy calculations to the study of semiconductors properties under high pressure has been reviewed in Ref. 60, showing that the phase stability, electronic and dynamical properties of compounds under pressure are well describe by DFT.

Furthermore, since the calculation of the disordered bcc phase is not possible to do with the VASP code, we have attempted to perform calculations for the bcc-like monoclinic $C 2 / m$ structure proposed in Ref. 34. Also, because the thermodynamic-phase transition between two structures occurs when the Gibbs free energy $(G)$ is the same for both phases, we have obtained the Gibbs free energy of the different phases using a quasiharmonic Debye model ${ }^{61}$ that allows obtaining $\mathrm{G}$ at room temperature from calculations performed for $T=0 \mathrm{~K}$ in order to discuss the relative stability of the different phases proposed in the present work.

In order to fully confirm whether the experimentally measured Raman scattering of the high-pressure phases of $\mathrm{Bi}_{2} \mathrm{Te}_{3}$ agree with theoretical estimates for these phases, we have also performed lattice-dynamics calculations of the phonon modes in the $R-3 m, C 2 / m$, and $C 2 / c$ phases at the zone center ( $\Gamma$ point) of the BZ. Our theoretical results enable us to assign the Raman modes observed for the different phases of $\mathrm{Bi}_{2} \mathrm{Te}_{3}$. Furthermore, the calculations also provide information about the symmetry of the modes and polarization vectors, which is not readily accessible in the present experiment. Highly converged results on forces are required for the calculation of the dynamical matrix. We use the direct-force constant approach (or supercell method). ${ }^{62}$ Highly converged results on forces are required for the calculation of the dynamical matrix. The construction of the dynamical matrix at the $\Gamma$ point of the $\mathrm{BZ}$ is particularly simple and involves separate calculations of the forces in which a fixed displacement from the equilibrium configuration of the atoms within the primitive unit cell is considered. Symmetry aids by reducing the number of such independent displacements, reducing the computational effort in the study of the analyzed structures considered in this work. Diagonalization of the dynamical matrix provides both the frequencies of the normal modes and their polarization vectors. It allows to us to identify the irreducible representation and the character of the phonon's modes at the $\Gamma$ point. In this work we provide and discuss the calculated frequencies and pressure coefficients of the Raman-active modes for the three calculated phases of $\mathrm{Bi}_{2} \mathrm{Te}_{3}$. The theoretical results obtained for infrared-active modes for the three calculated phases of $\mathrm{Bi}_{2} \mathrm{Te}_{3}$ are given as supplementary material of this article. $^{63}$
Finally, we want to mention that we have also checked the effect of the SO coupling in the structural stability and the phonon frequencies of the different phases. We have found that the effect of the SO coupling is very small and did not affect our present results (small differences of $1-3 \mathrm{~cm}^{-1}$ in the phonon frequencies at the $\Gamma$ point) but increased substantially the computer time so that the cost of the computation was very high for the more complex monoclinic high-pressure phases, as already discussed in Ref. 34. Therefore, all the theoretical values corresponding to lattice-dynamics calculations in the present paper do not include the SO coupling. In order to test our calculations, we show in Table I the calculated lattice parameters in the different phases of $\mathrm{Bi}_{2} \mathrm{Te}_{3}$ at different pressures. For the sake of comparison we show in Table I other theoretical calculations and experimental results available. As far as the $R-3 m$ phase is concerned, our calculated lattice parameters are in relatively good agreement with experimental values from Refs. 6 and 36. Our calculations with GGAPBEsol give values which are intermediate between those calculated with GGA-PBE and local density approximation (LDA), as it is generally known. Additionally, we give the calculated lattice parameters of $\mathrm{Bi}_{2} \mathrm{Te}_{3}$ in the monoclinic $C 2 / \mathrm{m}$ and $C 2 / c$ structures at 7.7 and $15.5 \mathrm{GPa}$, respectively, for comparison with experimental data. Note that in Table I the $a$ and $b$ lattice parameters of the $C 2 / m$ and $C 2 / c$ structures at 7.7 and $15.5 \mathrm{GPa}$ are very similar to those reported by Zhu et al. $;^{34}$ however, the $c$ lattice parameter and $\beta$ angle for monoclinic $C 2 / m$ and $C 2 / c$ structures differ from those obtained by Zhu et al. ${ }^{34}$ The reason is the results of our $a b$ initio calculations are given in the standard setting for the monoclinic structures, in contrast with Ref. 34, for a better comparison to future experiments since many experimentalists use the standard setting.

\section{RESULTS AND DISCUSSION}

\section{A. Optical absorption of $\alpha-\mathrm{Bi}_{2} \mathrm{Te}_{3}$ under pressure}

It is known that $\alpha-\mathrm{Bi}_{2} \mathrm{Te}_{3}$ has an indirect forbidden bandgap, $E_{\text {gap }}$, between 130 and $170 \mathrm{meV} \cdot{ }^{19,64-66}$ Figure 1 shows the optical transmittance of our $\alpha-\mathrm{Bi}_{2} \mathrm{Te}_{3}$ sample in the mid-IR region at room pressure outside the DAC. The spectrum near the fundamental absorption edge is dominated by large interferences. The large amplitude of the interference fringe pattern in the transparent region is a result of the high value of the refractive index, that is larger than $9 .{ }^{42,65,66}$ The sample transmittance and the interference-fringe amplitude decreases at low-photon energy due to the onset of free-carrier absorption and to high energies due to the fundamental absorption edge caused by band-to-band absorption. The absorption coefficient can be accurately determined from the transmittance spectrum only in a small photon energy range between the end of the interference pattern and the photon energy at which the transmitted intensity merges into noise. In this interval the absorption coefficient exhibits an exponential dependence on the photon energy. This prevents a detailed analysis of the absorption edge shape. Consequently, the optical bandgap has been determined by fitting a calculated transmittance to the experimental one. We calculate the transmittance by assuming 
TABLE I. Calculated (th.) and experimental (exp.) lattice parameters, bulk modulus $\left(\mathrm{B}_{0}\right)$, and its derivative $\left(\mathrm{B}_{0}{ }^{\prime}\right)$ of $\mathrm{Bi}_{2} \mathrm{Te}_{3}$ in the $R-3 m$ structure at ambient pressure and calculated lattice parameters of $\mathrm{Bi}_{2} \mathrm{Te}_{3}$ in the $C 2 / m$ and $C 2 / c$ structures at 8.4 and $15.5 \mathrm{GPa}$, respectively.

\begin{tabular}{|c|c|c|c|c|c|c|c|}
\hline & $\mathrm{a}(\AA)$ & $\mathrm{b}(\AA)$ & $c(\AA)$ & $\beta(\infty)$ & $\mathrm{B}_{0}(\mathrm{GPa})$ & $\mathrm{B}_{0}^{\prime}$ & Ref. \\
\hline \multicolumn{8}{|l|}{$\alpha-\mathrm{Bi}_{2} \mathrm{Te}_{3}(0 \mathrm{GPa})$} \\
\hline th.(GGA-PBEsol) & 4.380 & & 29.982 & & 41.92 & 4.89 & This work \\
\hline th. (GGA-PBESol) ${ }^{\mathrm{a}}$ & 4.375 & & 30.167 & & 41.61 & 4.68 & This work \\
\hline th.(GGA-PBE) & 4.45 & & 31.63 & & & & 49 \\
\hline th. $(\text { GGA-PBE) })^{\mathrm{a}}$ & 4.47 & & 31.12 & & & & 49 \\
\hline th. $(\mathrm{LDA})^{\mathrm{a}}$ & 4.36 & & 30.38 & & & & 47 \\
\hline exp. & 4.385 & & 30.497 & & & & 6 \\
\hline \multirow[t]{2}{*}{ exp. } & 4.383 & & 30.380 & & $32.5^{\mathrm{b}}$ & $10.1^{\mathrm{b}}$ & 36 \\
\hline & & & & & $40.9^{c}$ & $3.2^{\mathrm{c}}$ & \\
\hline \multicolumn{8}{|l|}{$\beta-\mathrm{Bi}_{2} \mathrm{Te}_{3}(8.4 \mathrm{GPa})$} \\
\hline th.(GGA-PBESol) & 14.883 & 4.066 & 9.121 & 89.73 & 41.25 & 4.06 & This work \\
\hline th. $(\mathrm{GGA}-\mathrm{PBE})^{\mathrm{d}}$ & 14.865 & 4.056 & 17.468 & 148.39 & & & 34 \\
\hline $\exp ^{\mathrm{d}}$ & 14.645 & 4.096 & 17.251 & 148.48 & & & 34 \\
\hline \multicolumn{8}{|l|}{$\gamma-\mathrm{Bi}_{2} \mathrm{Te}_{3}(15.5 \mathrm{GPa})$} \\
\hline th.(GGA-PBESol) & 9.895 & 6.962 & 7.709 & 70.30 & 45.28 & 3.57 & This work \\
\hline th. $(\text { GGA-PBE })^{\mathrm{e}}$ & 9.956 & 7.146 & 10.415 & 134.86 & & & 34 \\
\hline exp. ${ }^{e}$ & 10.233 & 6.955 & 10.503 & 136.0 & & & 34 \\
\hline
\end{tabular}

${ }^{\mathrm{a}}$ Calculations including the SO coupling.

${ }^{\mathrm{b}}$ At room pressure.

${ }^{c}$ Above $3.2 \mathrm{GPa}$.

${ }^{\mathrm{d}}$ Around 12-12.6 GPa.

eAround 14-14.4 GPa.

an absorption coefficient with two terms

$$
\alpha(E)=\frac{A}{E^{2}}+B e^{-\frac{E \text { gap }-E}{\Gamma}}
$$

where the first one corresponds to the free-carrier contribution and the second one corresponds to the Urbach tail of the fundamental absorption edge. Equation (1) was used to fit the calculated transmittance spectra to the experimental ones. The dotted line in Fig. 1 was calculated with Equation (1) by using only $A$ and $E_{\text {gap }}$ as fitting parameters, where $E_{\text {gap }}=$ $159 \mathrm{meV}$ at room pressure.

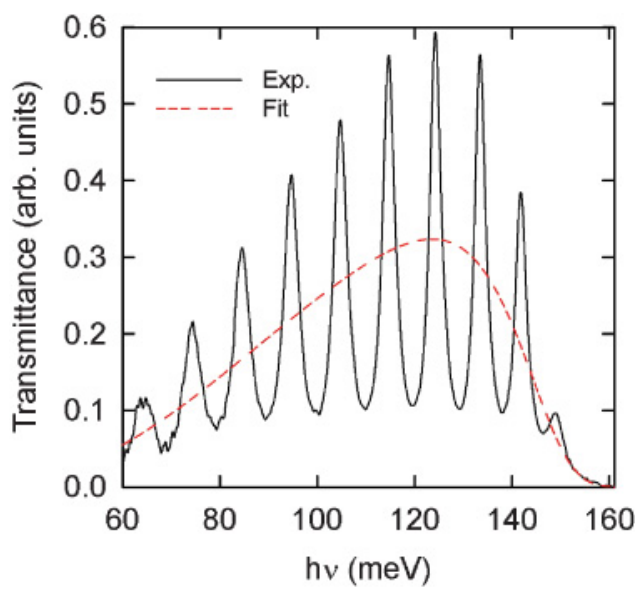

FIG. 1. (Color online) Experimental transmittance of a 7- $\mu \mathrm{m}$ thick $\alpha-\mathrm{Bi}_{2} \mathrm{Te}_{3}$ sample at room pressure outside the DAC (solid line). Dotted line indicates the fit of the experimental spectrum.
Figure 2 shows the $\mathrm{Bi}_{2} \mathrm{Te}_{3}$ transmittance spectrum for several pressures up to $5.5 \mathrm{GPa}$. Above that pressure the signal-to-noise ratio is too low to determine the optical bandgap energy. Figure 3 shows the pressure dependence of the optical bandgap of $\mathrm{Bi}_{2} \mathrm{Te}_{3}$, as determined from the previously described procedure. The pressure coefficient turns out to be $-6.4 \pm 0.6 \mathrm{meV} / \mathrm{GPa}$. This pressure coefficient of the optical bandgap is close to the value we obtained for the pressure dependence of the indirect bandgap from $a b$ initio calculations $(-10 \mathrm{meV} / \mathrm{GPa})$. From this result it appears that, even if the sample becomes opaque at 5.5 $\mathrm{GPa}, \mathrm{Bi}_{2} \mathrm{Te}_{3}$ still has a finite bandgap of some $120 \mathrm{meV}$.

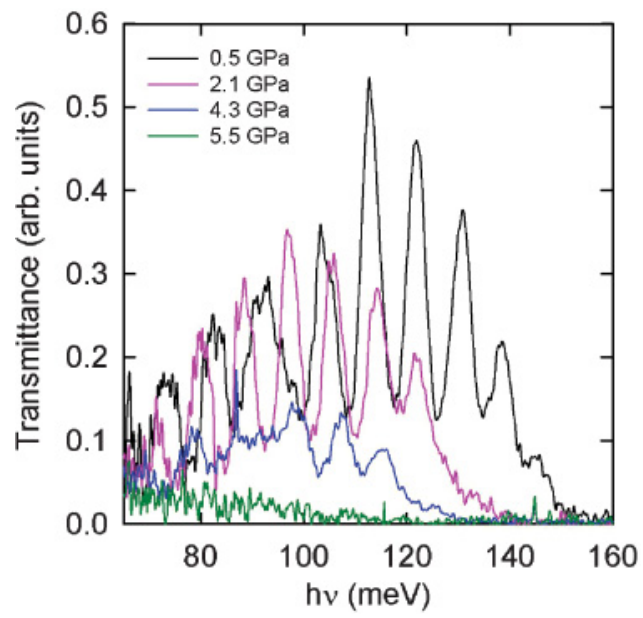

FIG. 2. (Color online) Experimental transmittance of $\alpha-\mathrm{Bi}_{2} \mathrm{Te}_{3}$ at different pressures up to 5.5. GPa. A shift of the absorption edge to low energies is observed with increasing pressure. 


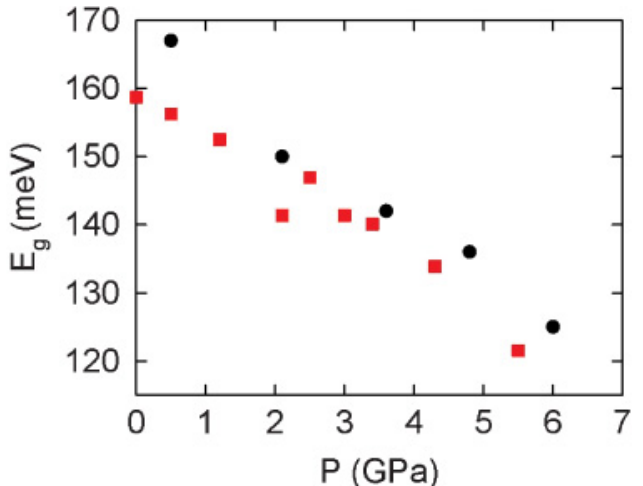

FIG. 3. (Color online) Pressure dependence of the optical bandgap of $\alpha-\mathrm{Bi}_{2} \mathrm{Te}_{3}$ according to reflectance (red squares) and to transmittance (black circles) measurements.

Sample opacity above 5.5 GPa seems to be then a result of the free-carrier absorption tail shifting to higher energies as the carrier concentration increases. Consequently, the sample opacity is likely caused by the overlap of the free-carrier absorption tail with the fundamental-absorption tail rather than a real closure of the bandgap. We have to note that our pressure coefficient of the optical bandgap is somewhat smaller in module than the pressure coefficient previously reported for the indirect bandgap: $-22 \mathrm{meV} / \mathrm{GPa}^{19} ;-12 \mathrm{meV} / \mathrm{GPa}$ below $3 \mathrm{GPa}$; and $-60 \mathrm{meV} / \mathrm{GPa}$ above $3 \mathrm{GPa}^{20}$ We have to consider that the estimation of these pressure coefficients in Refs. 19 and 20 were indirectly obtained from the pressure dependence of the electrical conductivity and those estimations suffer considerable errors since they assume that the change in resistivity is only due to the change of the indirect bandgap energy, which is not a well-founded assumption in extrinsic degenerate semiconductors.

In order to confirm our results on optical absorption we have performed high-pressure reflectance measurements in a 3- $\mu \mathrm{m}-$ thick sample whose results are shown in Fig. 4. The reflectance spectrum also exhibits a large interference fringe pattern in the transparency region, with amplitude decreasing to low and high photon energies. The reflectance spectrum at $6 \mathrm{GPa}$

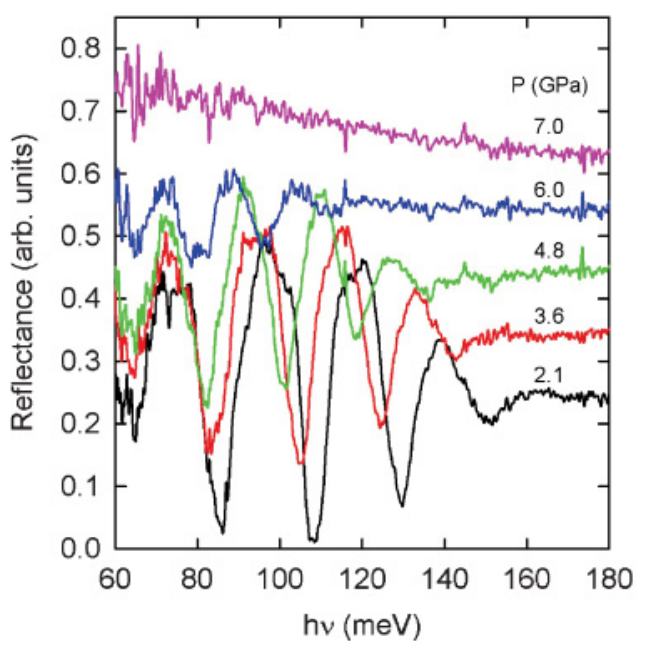

FIG. 4. (Color online) Experimental reflectance of $\alpha-\mathrm{Bi}_{2} \mathrm{Te}_{3}$ at different pressures. shows that the sample exhibits a clear onset of the fundamental absorption edge at around $120 \mathrm{meV}$ and also that the freecarrier absorption edge, even if it has shifted to higher energies, has not overlapped the fundamental absorption. Therefore our reflectance measurements allow us to confirm the results obtained from absorption measurements. Furthermore, the bandgap pressure coefficient, as determined from the shift of the photon energy at which interferences disappear, agrees with the one determined from the transmission spectra. At $7 \mathrm{GPa}$, a clear change in the reflectance occurs, with a large increase of the reflectance by $80 \%$ in the low-energy range. A large reflectance minimum (not shown here) appears at some $4000 \mathrm{~cm}^{-1}(500 \mathrm{meV})$, suggesting a phase transition to a metallic phase. The metallic nature of the high-pressure phases is in good agreement with previously reported resistivity measurements. ${ }^{17,21,28-30}$ If the reflectance minimum is taken as an estimation of the plasma frequency of the high-pressure phase above $7 \mathrm{GPa}$, the carrier concentration would be larger than $10^{21} \mathrm{~cm}^{-3}$ (assuming the same dielectric constant as in the rhombohedral phase). If the dielectric constant in $\beta$ phase is much smaller, the carrier concentration should be close to $10^{22} \mathrm{~cm}^{-3}$, which is more consistent with the observed superconducting behavior. ${ }^{28-30}$

The shift of the free-carrier absorption tail follows the increase of the free-carrier plasma frequency. Then the pressure dependence of the plasma frequency can be estimated from the shift of the photon energy at which the free-carrier absorption tail quenches the interference fringe pattern. Reflectance measurements outside the cell show that the plasma frequency at ambient pressure is below $50 \mathrm{meV}$, consistently with the hole concentration that is of the order of $7 \cdot 10^{18} \mathrm{~cm}^{-3}$, as measured by Hall effect. At $4.3 \mathrm{GPa}$ interference fringes are observed down to some $60 \mathrm{meV}\left(560 \mathrm{~cm}^{-1}\right)$. This upper limit to the plasma frequency would correspond to hole concentration of lower than $10^{19} \mathrm{~cm}^{-3}$, typical of a degenerate semiconductor.

This increase in the hole concentration should result in a Burstein-Moss positive contribution to the optical bandgap, which explains the discrepancy between the experimental and theoretical value of the bandgap pressure coefficient. The bandgap around $5 \mathrm{GPa}$ is in fact smaller than the measured optical gap. Given the band structure of $\mathrm{Bi}_{2} \mathrm{Te}_{3},{ }^{67}$ with six equivalent minima in the valence band, the density of states is very large and the hole concentration per minimum would be only of some $1.5 \times 10^{18} \mathrm{~cm}^{-3}$, which would lead to a Burstein-Moss shift of some $50 \mathrm{meV}$ for a hole effective mass of $0.09 \mathrm{~m}_{0} .{ }^{68}$ Then even taking into account the Burstein-Moss shift, $\mathrm{Bi}_{2} \mathrm{Te}_{3}$ at $5 \mathrm{GPa}$ would still be a low-gap semiconductor. In fact this estimation of the Burstein-Moss shift is based on the ambient-pressure electronic structure. At pressures above the ETT transition the density of states in the valence-band maximum is expected to be much larger as the ellipsoids merge into a thoroidal ring, as proposed by Istkevitch et al. ${ }^{69}$ Consequently, the Burstein-Moss shift above the ETT should be much lower than $50 \mathrm{meV}$.

Finally, we must note that our analysis of the optical absorption edge in $\mathrm{Bi}_{2} \mathrm{Te}_{3}$ have not allowed us to detect any change in the pressure dependence of the indirect bandgap around $3 \mathrm{GPa}$ to confirm the presence of an ETT as observed in other works..$^{20,29,31,32,35,36}$ The very small change in the pressure coefficient of the indirect bandgap seems not to 


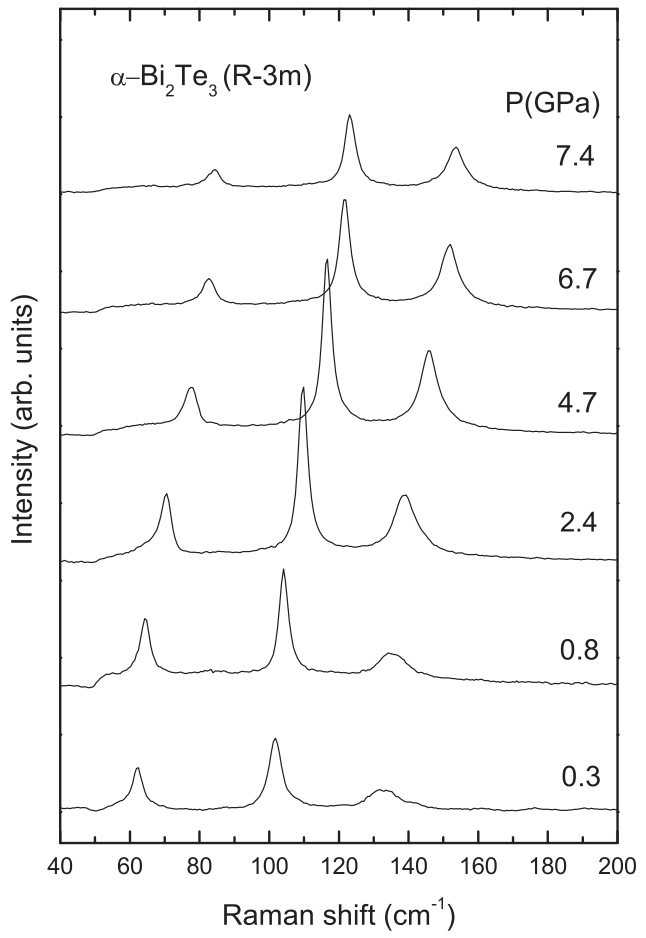

FIG. 5. Experimental Raman spectra of $\alpha-\mathrm{Bi}_{2} \mathrm{Te}_{3}$ at pressures between room pressure and 7.4 GPa.

be affected by the ETT since there is no change in volume but in volume compressibility, and the change is very subtle to be measured in our transmission or reflection spectra in comparison with the drastic effects observed in transport measurements or even in the parameters of the Raman modes (as will be discussed in the next section).

\section{B. Raman scattering of $\alpha-\mathrm{Bi}_{2} \mathrm{Te}_{3}$ under pressure}

The rhombohedral structure of $\alpha-\mathrm{Bi}_{2} \mathrm{Te}_{3}$ is a centrosymmetric structure that has a primitive cell with one Te atom located in a 3a Wyckoff position and the remaining $\mathrm{Bi}(2)$ and $\mathrm{Te}(2)$ atoms occupying 6c Wyckoff sites. Therefore, group theory allows 10 zone-center modes, which decompose in the irreducible representations as follows ${ }^{70}$ :

$$
\Gamma_{10}=2 A_{1 \mathrm{~g}}+3 A_{2 \mathrm{u}}+2 E_{\mathrm{g}}+3 E_{\mathrm{u}} .
$$

The two acoustic branches come from one $\mathrm{A}_{2 \mathrm{u}}$ and a doubly degenerated $E_{\mathrm{u}}$ mode, while the rest correspond to optic modes. Gerade $(\mathrm{g})$ modes are Raman active while ungerade (u) modes are infrared (IR) active. Therefore, there are four Raman-active modes $\left(2 A_{1 \mathrm{~g}}+2 E_{\mathrm{g}}\right)$ and four IR-active modes $\left(2 A_{2 \mathrm{u}}+2 E_{\mathrm{u}}\right)$. The $E_{\mathrm{g}}$ modes correspond to atomic vibrations in the plane of the layers, while the $A_{1 \mathrm{~g}}$ modes correspond to vibrations along the $c$ axis perpendicular to the layers. ${ }^{42-44,50}$

Figure 5 shows the experimental Raman spectra of $\alpha-\mathrm{Bi}_{2} \mathrm{Te}_{3}$ at different pressures up to $7.4 \mathrm{GPa}$. We have observed and followed under pressure three out of the four Raman-active modes. The $E_{\mathrm{g}}$ mode calculated to be close to $40 \mathrm{~cm}^{-1}$ has not been observed in our experiments as it was also not seen in previous Raman-scattering measurements at room and high pressures. ${ }^{42,50,71-73}$ Figure 6(a) shows the experimentalpressure dependence of the frequencies of the three first-order
Raman modes measured in $\alpha-\mathrm{Bi}_{2} \mathrm{Te}_{3}$, and Table II summarizes our experimental and theoretical first-order Raman-mode frequencies and pressure coefficients in the rhombohedral phase. Our experimental frequencies at room pressure are in good agreement with those already measured in Ref. 42 and Ref. 50 and with those recently measured in Refs. 45 and 71-73. On the other hand our theoretical frequencies at room pressure are also in good agreement with those reported in Ref. 49 without SO coupling (see Table II) and are slightly larger than those calculated including SO coupling (see Ref. 49).

In Fig. 6(a) it can be observed that all the measured Raman modes exhibit a hardening with increasing pressure. The experimental values of the pressure coefficients of the Raman-mode frequencies are in a general good agreement with our theoretical calculations and with the values reported in Ref. 50 up to $0.5 \mathrm{GPa}$; however, a decrease of the pressure coefficient of two modes around $4.0 \mathrm{GPa}$ should be noted [see dashed lines in Fig. 6(b)]. We have attributed the less positive pressure coefficient of these two Raman modes to the pressureinduced ETT observed in $\mathrm{Sb}_{2} \mathrm{Te}_{3}$ and $\mathrm{Bi}_{2} \mathrm{Te}_{3} .{ }^{20,29,31,32,35,36} \mathrm{In}$ fact in a previous study in $\mathrm{Sb}_{2} \mathrm{Te}_{3}$ under pressure we have found a change in the pressure coefficient of the frequency of all modes measured.$^{51}$ In order to support our hypothesis we also plot as Fig. 6(b) the pressure dependence of the full width at half maximum (FWHM) of the three measured Raman modes. Curiously, it is observed that the FWHM changes its slope around $4 \mathrm{GPa}$ thus confirming an anomaly related to the ETT. Therefore, both our results of the pressure dependence of the frequency and linewidth give support to the observation of the ETT around 4.0 GPa in $\alpha-\mathrm{Bi}_{2} \mathrm{Te}_{3}$ similarly to the case of $\alpha-\mathrm{Sb}_{2} \mathrm{Te}_{3}{ }^{51}$

As previously commented, anomalies in the phonon spectrum are also expected for materials undergoing a ETT and have been observed in $\mathrm{Sb}_{1.5} \mathrm{Bi}_{0.5} \mathrm{Te}_{3}{ }^{15}$ In the latter work the high-frequency $A_{1 \mathrm{~g}}$ mode was not altered near the ETT in good agreement with our measurements; however, we have noted a change both in the lower $A_{1 \mathrm{~g}}$ and the higher-frequency $E_{\mathrm{g}}$ modes. Since $A_{1 \mathrm{~g}}$ modes are polarized in the direction perpendicular to the layers while the $E_{\mathrm{g}}$ modes are polarized along the layers, our observation of a less positive pressure coefficient at $4.0 \mathrm{GPa}$ of both modes in $\alpha-\mathrm{Bi}_{2} \mathrm{Te}_{3}$ suggests that the ETT in $\mathrm{Bi}_{2} \mathrm{Te}_{3}$ is related to a change of the structural compressibility of both the direction perpendicular to the layers and the direction along the layers. This seems not to be in agreement with Polian et al.'s observations, which suggest that the ETT in $\mathrm{Bi}_{2} \mathrm{Te}_{3}$ only affects the plane of the layers. ${ }^{36}$ Consequently, more work is needed to understand the mechanism of the ETT in this material.

To conclude this section regarding the rhombohedral structure of $\alpha-\mathrm{Bi}_{2} \mathrm{Te}_{3}$, we want to make a comment on the pressure coefficients of the Raman modes of this structure in comparison to those recently measured in $\alpha-\mathrm{Sb}_{2} \mathrm{Te}_{3} \cdot{ }^{51}$ It is known that in chalcogenide laminar materials, the two lowest frequency $E$ and $A$ modes are usually related to shear vibrations between adjacent layers along the $a-b$ plane and to vibrations of one layer against the others along the $c$ axis, respectively. It has been commented that the $E$ mode displays the smallest pressure coefficient due to the weak bending force constant between the interlayer distances (in our case, Te-Te distances) while 

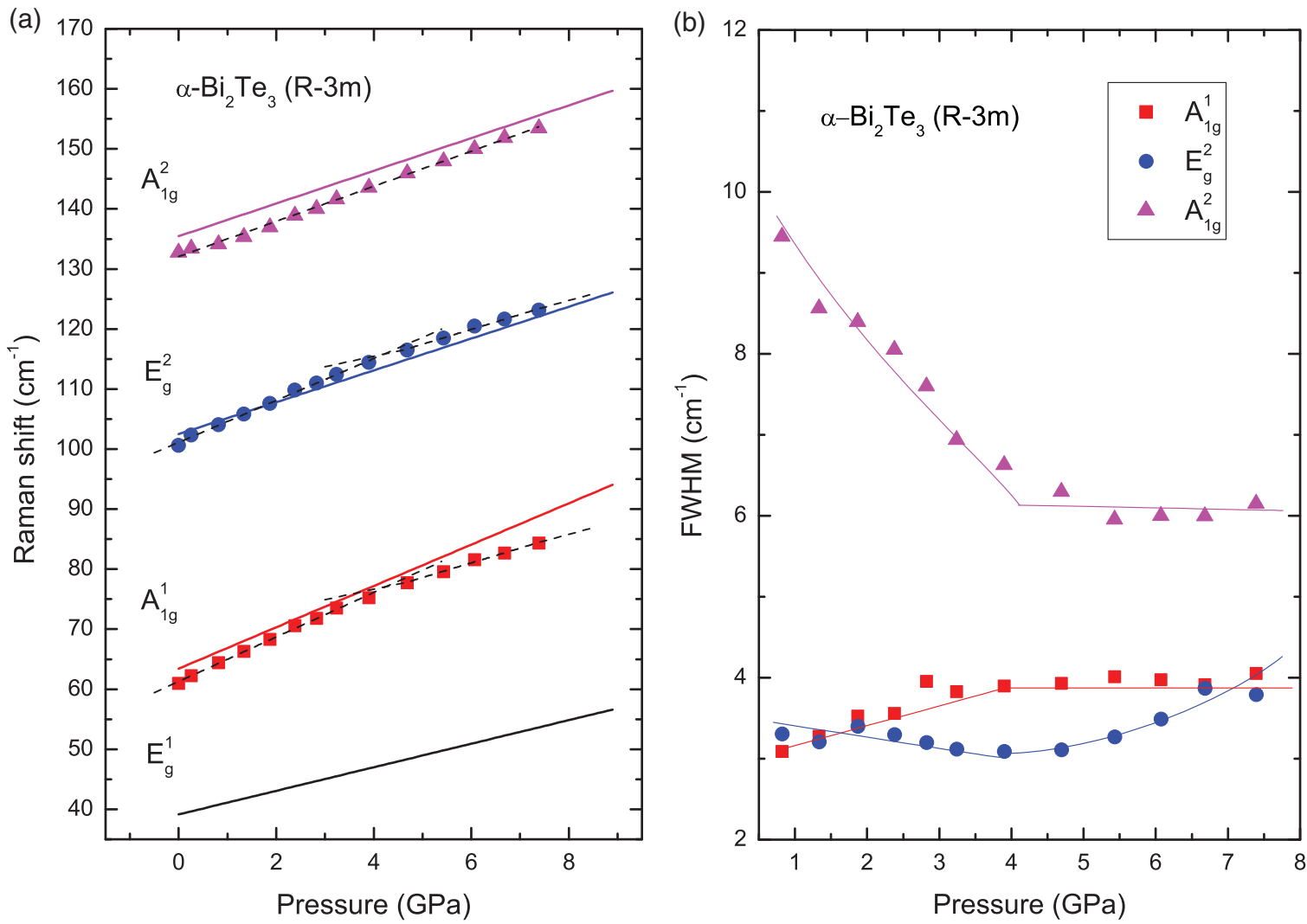

FIG. 6. (Color online) (a) Experimental pressure dependence of the Raman mode frequencies in $\alpha-\mathrm{Bi}_{2} \mathrm{Te}_{3}$. Solid curves represent $a b$ initio calculated-mode frequencies. Dashed lines are guides to the eye to indicate the different behavior of the experimental Raman mode frequencies with pressure. (b) Experimental pressure dependence of the FWHM of the Raman modes. Solid lines are guide to the eye to indicate the different behavior of the FWHM with pressure.

the $A$ mode displays the largest pressure coefficient due to the extraordinary increase of the stretching force constant between the interlayer distances. ${ }^{51}$ For example, the $E$ and $A$ modes with frequencies around $40(60) \mathrm{cm}^{-1}$ and $116(133) \mathrm{cm}^{-1}$ in InSe $(\mathrm{GaSe})$, respectively, have pressure coefficients of $0.68(0.85) \mathrm{cm}^{-1} / \mathrm{GPa}$ and $5.41(5.78) \mathrm{cm}^{-1} / \mathrm{GPa}^{74,75} \mathrm{In}$ $\alpha-\mathrm{Bi}_{2} \mathrm{Te}_{3}$ our theoretical calculations show that the two lowestfrequency $A_{1 \mathrm{~g}}$ and $E_{\mathrm{g}}$ modes have rather similar pressure coefficients thus suggesting that this compound does not have a strong anisotropy in their intralayer and interlayer properties as other layered chalcogenides. We arrived at a similar conclusion in our study of $\alpha-\mathrm{Sb}_{2} \mathrm{Te}_{3},{ }^{51}$ so we attribute the stronger interlayer interaction occurring in $\mathrm{Sb}_{2} \mathrm{Te}_{3}$ and $\mathrm{Bi}_{2} \mathrm{Te}_{3}$ to the effect of the SO coupling, which is absent in other laminar chalcogenides such as InSe and GaSe. In regard to the values of the pressure coefficients of these modes, they

TABLE II. Experimental room-temperature Raman-mode frequencies and pressure coefficients observed in $\alpha$ - $\mathrm{Bi}_{2} \mathrm{Te}_{3}$ at both room pressure and $4.0 \mathrm{GPa}$, as obtained from fits to the data using $\omega(P)=\omega\left(P_{0}=1 \mathrm{~atm}\right)+a_{1} \cdot\left(P-P_{0}\right)$ and $\omega(P)=\omega\left(\mathrm{P}_{0}=4 \mathrm{GPa}\right)+a_{1} \cdot\left(P-P_{0}\right)$, respectively. Theoretical (th.) values calculated at room pressure are also shown for comparison, as well as theoretical and experimental data from Refs. 49 and 50, respectively.

\begin{tabular}{|c|c|c|c|c|c|c|c|}
\hline Mode & $\begin{array}{c}\omega\left(P=P_{0}\right) \\
\quad\left(\mathrm{cm}^{-1}\right)\end{array}$ & $a_{1}\left(\mathrm{~cm}^{-1} / \mathrm{GPa}\right)$ & $\begin{array}{l}\omega\left(P=P_{0}\right) \\
\quad\left(\mathrm{cm}^{-1}\right)\end{array}$ & $a_{1}\left(\mathrm{~cm}^{-1} / \mathrm{GPa}\right)$ & $\begin{array}{c}\omega\left(P=P_{0}\right) \\
\text { (th.) }\left(\mathrm{cm}^{-1}\right)\end{array}$ & $\begin{array}{c}a_{1}(\text { th. }) \\
\left(\mathrm{cm}^{-1} / \mathrm{GPa}\right)\end{array}$ & $\begin{array}{l}\omega\left(P=P_{0}\right) \\
\text { (th.) }\left(\mathrm{cm}^{-1}\right)\end{array}$ \\
\hline$E_{\mathrm{g}}^{1}$ & & & & & 39.2 & 1.96 & 36.4 \\
\hline \multirow[t]{2}{*}{$A_{1 \mathrm{~g}}^{1}$} & $61.3^{\mathrm{a}}$ & $3.71^{\mathrm{a}}$ & 62 & 3.4 & 63.4 & 3.44 & 53.9 \\
\hline & $76.1^{b}$ & $2.45^{\mathrm{b}}$ & & & & & \\
\hline \multirow[t]{2}{*}{$E_{\mathrm{g}}^{2}$} & $101.1^{\mathrm{a}}$ & $3.49^{\mathrm{a}}$ & 102 & 4.0 & 102.5 & 2.65 & 104.4 \\
\hline & $115.1^{\mathrm{b}}$ & $2.48^{\mathrm{b}}$ & & & & & \\
\hline$A_{1 \mathrm{~g}}^{2}$ & $132.1^{\mathrm{a}}$ & $2.92^{\mathrm{a}}$ & & & 135.5 & 2.72 & 137.2 \\
\hline Ref. & $*$ & $*$ & 50 & 50 & $*$ & $*$ & 49 \\
\hline
\end{tabular}

${ }^{\mathrm{a}}$ Calculated at room pressure $\left(P_{0}=1 \mathrm{~atm}\right)$.

${ }^{\mathrm{b}}$ Calculated at $P_{0}=4.0 \mathrm{GPa}$.

*This work. 
are slightly smaller for $\mathrm{Bi}_{2} \mathrm{Te}_{3}$ than for $\mathrm{Sb}_{2} \mathrm{Te}_{3}$. On the opposite end the other two Raman modes with higher frequency exhibit larger pressure coefficients in $\mathrm{Bi}_{2} \mathrm{Te}_{3}$ than in $\mathrm{Sb}_{2} \mathrm{Te}_{3}$. The larger pressure coefficients of the higher-frequency modes in $\mathrm{Bi}_{2} \mathrm{Te}_{3}$ than in $\mathrm{Sb}_{2} \mathrm{Te}_{3}$, which are mainly related to intralayer vibrations, suggest that the intralayer bonds are more covalent in $\mathrm{Bi}_{2} \mathrm{Te}_{3}$ than in $\mathrm{Sb}_{2} \mathrm{Te}_{3}$. On the contrary the smaller pressure coefficients of the lower-frequency modes in $\mathrm{Bi}_{2} \mathrm{Te}_{3}$ than in $\mathrm{Sb}_{2} \mathrm{Te}_{3}$, which are more influenced by interlayer vibrations, suggest that the interlayer bonds of van der Waals type tend to harden at a faster rate with pressures in $\mathrm{Sb}_{2} \mathrm{Te}_{3}$ than in $\mathrm{Bi}_{2} \mathrm{Te}_{3}$, i.e., the anisotropy in the properties along the layers and perpendicular to the layers tend to disappear more quickly with increasing pressure in $\mathrm{Sb}_{2} \mathrm{Te}_{3}$ than in $\mathrm{Bi}_{2} \mathrm{Te}_{3}$.

\section{Raman scattering of $\beta-\mathrm{Bi}_{2} \mathrm{Te}_{3}$ under pressure}

It has been recently demonstrated in a joint experimental and theoretical work that $\alpha-\mathrm{Bi}_{2} \mathrm{Te}_{3}$ undergoes a phase transition around 7.5 GPa toward a $\beta-\mathrm{Bi}_{2} \mathrm{Te}_{3}$ phase with monoclinic $C 2 / m$ structure. ${ }^{34}$ Figure 7 (a) shows the experimental Raman spectra of $\beta-\mathrm{Bi}_{2} \mathrm{Te}_{3}$ at different pressures from $8.4 \mathrm{GPa}$ up to $14.4 \mathrm{GPa}$. In the monoclinic $C 2 / \mathrm{m}$ structure, all $\mathrm{Bi}$ and $\mathrm{Te}$ atoms occupy $4 \mathrm{i}$ Wyckoff sites. ${ }^{34}$ Therefore, group theoretical considerations predict 30 vibrational modes with the following representation, ${ }^{70}$

$$
\Gamma_{30}=\left(10 A_{\mathrm{g}}+10 B_{\mathrm{u}}\right)+\left(5 B_{\mathrm{g}}+5 A_{\mathrm{u}}\right) .
$$

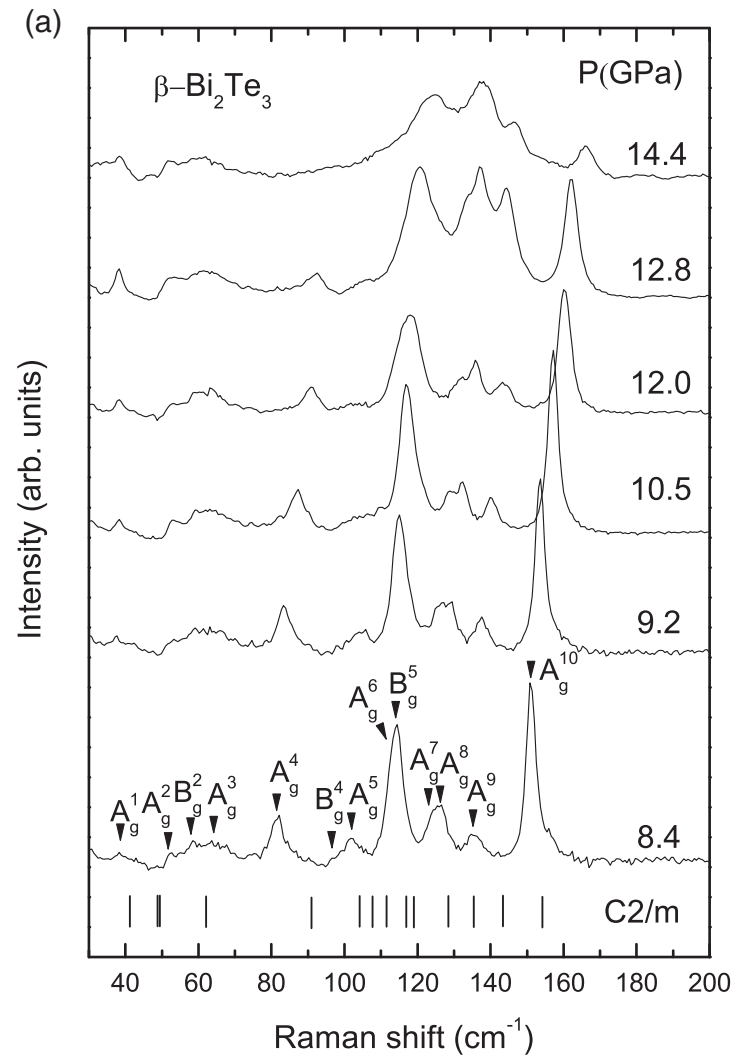

From them, one $A_{\mathrm{u}}$ and two $B_{\mathrm{u}}$ are the acoustic phonons and the rest are optical phonons. Consequently, we expect 15 zonecenter Raman-active $\left(10 A_{\mathrm{g}}+5 B_{\mathrm{g}}\right)$ modes for the $C 2 / \mathrm{m}$ phase. For the sake of comparison we have marked at the bottom of Fig. 7(a) the calculated Raman-mode frequencies for this phase at $8.4 \mathrm{GPa}$ to compare it with the experimental Raman spectrum at $8.4 \mathrm{GPa}$. It can be observed that the frequencies of the experimental Raman modes agree reasonably with our calculations, but most of the high-frequency modes show smaller experimental frequencies (around $6 \mathrm{~cm}^{-1}$ on average) than suggested by the calculations. Table III summarizes the experimental and theoretical first-order Raman-mode frequencies and pressure coefficients at $8.4 \mathrm{GPa}$ in $\beta-\mathrm{Bi}_{2} \mathrm{Te}_{3}$. A fit of the experimental Raman spectrum at $8.4 \mathrm{GPa}$ to Voigt profiles is reported in the supplementary material. ${ }^{63}$ Figure 7(b) shows the pressure dependence of the experimental and theoretical Raman-mode frequencies in $\beta-\mathrm{Bi}_{2} \mathrm{Te}_{3}$. It can be observed that there is a rather nice agreement between the experimental and theoretical Raman-mode pressure coefficients. Therefore, we can reasonably confirm that the $\beta-\mathrm{Bi}_{2} \mathrm{Te}_{3}$ phase has the monoclinic $C 2 / m$ structure already found in $\mathrm{Bi}_{2} \mathrm{Te}_{3}$ by means of powder X-ray diffraction measurements. ${ }^{34}$

In our experiments we have not observed the lowestfrequency mode $B_{\mathrm{g}}^{1}$. Their detection is difficult because it must be a weak intensity mode deriving from the nonobserved $E_{\mathrm{g}}^{1}$ mode of $\alpha-\mathrm{Bi}_{2} \mathrm{Te}_{3}$ phase. On the other hand the detection of the $B_{\mathrm{g}}^{3}$ mode is difficult because it must be very weak and

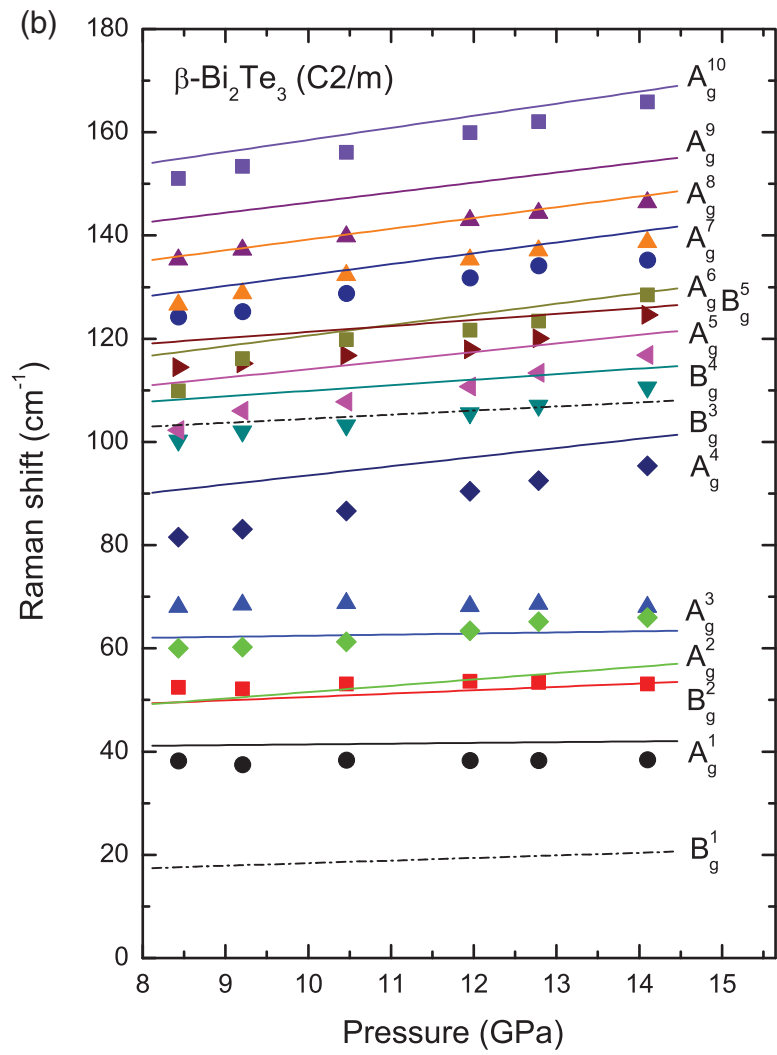

FIG. 7. (Color online) (a) Experimental Raman spectra of the high-pressure phases of $\beta$ - $\mathrm{Bi}_{2} \mathrm{Te}_{3}$ at pressures between 8.4 and $14.4 \mathrm{GPa}$. Bottom marks indicate the calculated frequencies of the Raman-active modes in the $\beta-\mathrm{Bi}_{2} \mathrm{Te}_{3}$ phase at $8.4 \mathrm{GPa}$. (b) Experimental pressure dependence of the Raman-mode frequencies in $\beta-\mathrm{Bi}_{2} \mathrm{Te}_{3}$. Solid (dashed-dotted) curves represent $a b$ initio calculated mode frequencies of modes observed (not observed) in our measurements. 
TABLE III. Experimental Raman-mode frequencies and pressure coefficients observed in $\beta-\mathrm{Bi}_{2} \mathrm{Te}_{3}$ at room temperature at $P_{0}=$ $8.4 \mathrm{GPa}$ as obtained from fits using $\omega(P)=\omega\left(P_{0}\right)+a_{1} \cdot\left(P-P_{0}\right)$. Theoretical (th.) ab initio values for the frequencies and pressure coefficients at $8.4 \mathrm{GPa}$ are also shown for comparison.

\begin{tabular}{lcccc}
\hline \hline Mode & $\begin{array}{c}\omega\left(P_{0}\right) \\
\left(\mathrm{cm}^{-1}\right)\end{array}$ & $a_{1}\left(\mathrm{~cm}^{-1} / \mathrm{GPa}\right)$ & $\begin{array}{c}\omega\left(P_{0}\right)(\mathrm{th} .) \\
\left(\mathrm{cm}^{-1}\right)\end{array}$ & $\begin{array}{c}\mathrm{a}_{1}(\mathrm{th} .) \\
\left(\mathrm{cm}^{-1} / \mathrm{GPa}\right)\end{array}$ \\
\hline $\mathrm{B}_{\mathrm{g}}^{1}$ & & & 17.6 & 0.50 \\
$\mathrm{~A}_{\mathrm{g}}^{1}$ & 38.0 & 0.06 & 41.1 & 0.14 \\
$\mathrm{~B}_{\mathrm{g}}^{2}$ & 52.4 & 0.18 & 49.5 & 0.65 \\
$\mathrm{~A}_{\mathrm{g}}^{2}$ & 60.0 & 1.16 & 49.6 & 1.23 \\
$\mathrm{~A}_{\mathrm{g}}^{3}$ & 68.4 & -0.09 & 62.1 & 0.21 \\
$\mathrm{~A}_{\mathrm{g}}^{4}$ & 81.4 & 2.49 & 90.7 & 1.77 \\
$\mathrm{~B}_{\mathrm{g}}^{3}$ & & & 103.2 & 0.79 \\
$\mathrm{~B}_{\mathrm{g}}^{4}$ & 100.1 & 1.69 & 108.2 & 1.08 \\
$\mathrm{~A}_{\mathrm{g}}^{5}$ & 102.9 & 2.39 & 111.4 & 1.65 \\
$\mathrm{~A}_{\mathrm{g}}^{6}$ & 112.1 & 2.84 & 117.3 & 2.05 \\
$\mathrm{~B}_{\mathrm{g}}^{5}$ & 113.7 & 1.63 & 119.4 & 1.16 \\
$\mathrm{~A}_{\mathrm{g}}^{7}$ & 124.2 & 2.09 & 128.9 & 2.11 \\
$\mathrm{~A}_{\mathrm{g}}^{8}$ & 127.1 & 2.17 & 135.9 & 2.09 \\
$\mathrm{~A}_{\mathrm{g}}^{9}$ & 135.6 & 1.97 & 143.2 & 1.95 \\
$\mathrm{~A}_{\mathrm{g}}^{10}$ & 151.0 & 2.56 & 154.7 & 2.34 \\
\hline \hline
\end{tabular}

may be degenerate with the $B_{\mathrm{g}}^{4}$ mode. Other weak modes have been observed at frequencies between 60 and $110 \mathrm{~cm}^{-1}$ at 8.4 GPa most of them forming part of broad bands. On the other hand the six Raman modes with highest frequencies above $100 \mathrm{~cm}^{-1}$ are considerably intense and dominate the Raman spectrum. The frequencies and pressure coefficients of these high-frequency modes are in good agreement with our calculations [see Fig. 7(b) and Table III] thus supporting our assignment of $\beta-\mathrm{Bi}_{2} \mathrm{Te}_{3}$ to the monoclinic $C 2 / m$ phase.

Finally, we want to make a comment on the $\mathrm{Bi}$ coordination of $\beta-\mathrm{Bi}_{2} \mathrm{Te}_{3}$. In this respect at the $R-3 m$ to $C 2 / m$ phase-transition pressure around $8.4 \mathrm{GPa}$ [see Fig. 7(a)], the highest-frequency mode of $\beta-\mathrm{Bi}_{2} \mathrm{Te}_{3}$ has a smaller frequency $\left(151 \mathrm{~cm}^{-1}\right)$ than the highest-frequency mode of $\alpha-\mathrm{Bi}_{2} \mathrm{Te}_{3}$ $\left(157 \mathrm{~cm}^{-1}\right)$. This decrease in frequency of the highestfrequency mode, usually related to stretching $\mathrm{Bi}$-Te vibrations, suggests an increase in the $\mathrm{Bi}-\mathrm{Te}$ distance related to an increase of the $\mathrm{Bi}$ coordination from sixfold in $\alpha-\mathrm{Bi}_{2} \mathrm{Te}_{3}$ to sevenfold in $\beta-\mathrm{Bi}_{2} \mathrm{Te}_{3}$. This result is in good agreement with the recently observed increase of the $\mathrm{Bi}$ coordination on going from $\alpha-\mathrm{Bi}_{2} \mathrm{Te}_{3}$ to $\beta-\mathrm{Bi}_{2} \mathrm{Te}_{3} .{ }^{34}$ Similar results have been already found in the study of the lattice dynamics of $\mathrm{Sb}_{2} \mathrm{Te}_{3}$ under pressure..$^{51}$

\section{Raman scattering of $\gamma-\mathrm{Bi}_{2} \mathrm{Te}_{3}$ and $\delta-\mathrm{Bi}_{2} \mathrm{Te}_{3}$ under pressure}

Similar to the previous case, it has been recently demonstrated in a joint experimental and theoretical work that $\beta-\mathrm{Bi}_{2} \mathrm{Te}_{3}$ undergoes a phase transition around $13.4 \mathrm{GPa}$ toward a $\gamma-\mathrm{Bi}_{2} \mathrm{Te}_{3}$ phase with monoclinic $C 2 / c$ structure. ${ }^{34}$ We have observed that the Raman spectrum above $15.5 \mathrm{GPa}$ is different from that of the $C 2 / m$ phase. Figure 8 (a) shows the experimental Raman spectra of $\gamma-\mathrm{Bi}_{2} \mathrm{Te}_{3}$ at different pressures from 15.5 GPa up to 22.0 GPa. Furthermore, it can be observed that the Raman spectrum disappears above $21.6 \mathrm{GPa}$ thus suggesting a phase transition to a Raman-inactive phase above this pressure. Additionally, on pressure release we have observed that the sample reverts back to the original rhombohedral phase below $5.0 \mathrm{GPa}$ after considerable hysteresis. The spectrum of the recovered sample in the rhombohedral phase at $1 \mathrm{~atm}$ after releasing pressure is shown as the last spectrum in the top of Fig. 8(a).

According to Zhu et al., Bi atoms occupy one $8 \mathrm{f}$ Wyckoff site, and Te atoms occupy one $8 \mathrm{f}$ and one $4 \mathrm{e}$ Wyckoff sites in the monoclinic $C 2 / c$ structure of $\gamma-\mathrm{Bi}_{2} \mathrm{Te}_{3} .{ }^{34}$ Consequently, group theoretical considerations predict 30 vibrational modes with the following representation, ${ }^{70}$

$$
\Gamma_{30}=\left(7 A_{\mathrm{g}}+7 A_{\mathrm{u}}\right)+\left(8 B_{\mathrm{g}}+8 B_{\mathrm{u}}\right) .
$$

One $A_{\mathrm{u}}$ and two $B_{\mathrm{u}}$ are acoustic modes and the rest are optical modes. Therefore, we expect 15 zone-center Ramanactive modes $\left(7 A_{\mathrm{g}}+8 B_{\mathrm{g}}\right)$ for the $C 2 / c$ phase.

For the sake of comparison we have marked in Fig. 8(a) the calculated Raman-mode frequencies for the $C 2 / c$ phase at 15.5 GPa to compare it with the Raman spectrum at $15.5 \mathrm{GPa}$. A fit of the experimental Raman spectrum at $15.5 \mathrm{GPa}$ to Voigt profiles is reported in the supplementary material. ${ }^{63}$ It can be observed that the frequencies of the experimental Raman modes agree reasonably well with our calculations at this pressure thus giving support to the assignment of the $\gamma-\mathrm{Bi}_{2} \mathrm{Te}_{3}$ to the $C 2 / c$ phase. Further, it can be noted that, at $15.5 \mathrm{GPa}$, the highest-frequency mode of $\gamma-\mathrm{Bi}_{2} \mathrm{Te}_{3}$ has a smaller frequency $\left(165 \mathrm{~cm}^{-1}\right)$ than the highest-frequency mode of $\beta-\mathrm{Bi}_{2} \mathrm{Te}_{3}$ $\left(170 \mathrm{~cm}^{-1}\right)$. This decrease in frequency of the highestfrequency mode again suggests an increase in the Bi-Te distance related to an increase of the $\mathrm{Bi}$ coordination from sevenfold in $\beta-\mathrm{Bi}_{2} \mathrm{Te}_{3}$ to eightfold in $\gamma-\mathrm{Bi}_{2} \mathrm{Te}_{3}$. This result is again in good agreement with the recently observed increase of the $\mathrm{Bi}$ coordination ongoing from $\beta-\mathrm{Bi}_{2} \mathrm{Te}_{3}$ to $\gamma-\mathrm{Bi}_{2} \mathrm{Te}_{3}{ }^{34}$ and also in recent results on $\mathrm{Sb}_{2} \mathrm{Te}_{3}$ under pressure. ${ }^{51}$

Figure 8(b) shows the pressure dependence of the experimental and theoretical Raman-mode frequencies in $\gamma-\mathrm{Bi}_{2} \mathrm{Te}_{3}$. It can be observed that there is a consistent agreement between the experimental and theoretical Raman-mode frequencies and pressure coefficients for this phase. Therefore, we can reasonably confirm that the $\gamma-\mathrm{Bi}_{2} \mathrm{Te}_{3}$ phase has the monoclinic $C 2 / c$ structure already found by means of x-ray diffraction measurements in $\mathrm{Bi}_{2} \mathrm{Te}_{3} .{ }^{34}$ Table IV summarizes the experimental and theoretical first-order Raman-mode frequencies at $15.5 \mathrm{GPa}$ and pressure coefficients in the $\gamma-\mathrm{Bi}_{2} \mathrm{Te}_{3}$ phase. In our experiments we have not observed the three lowest frequency modes $\left(B_{\mathrm{g}}^{1}, A_{\mathrm{g}}^{1}\right.$, and $\left.B_{\mathrm{g}}^{2}\right)$. Similarly to the previous case, their detection is difficult because they must be weakintensity modes deriving from the nonobserved $E_{\mathrm{g}}^{1}$ mode of $\alpha-\mathrm{Bi}_{2} \mathrm{Te}_{3}$ phase. On the other hand we have managed to follow the weak modes $A_{\mathrm{g}}^{2}, B_{\mathrm{g}}^{3}$, and $A_{\mathrm{g}}^{3}$ in the region between 60 and $80 \mathrm{~cm}^{-1}$. The next six modes between 100 and $150 \mathrm{~cm}^{-1}$ form a broad band from which we have decomposed six bands that increase in broadening from 15.5 to $19.8 \mathrm{GPa}$ [see Figs. 8(a) and 8(b)], with $A_{\mathrm{g}}^{6}$ being the weakest mode 

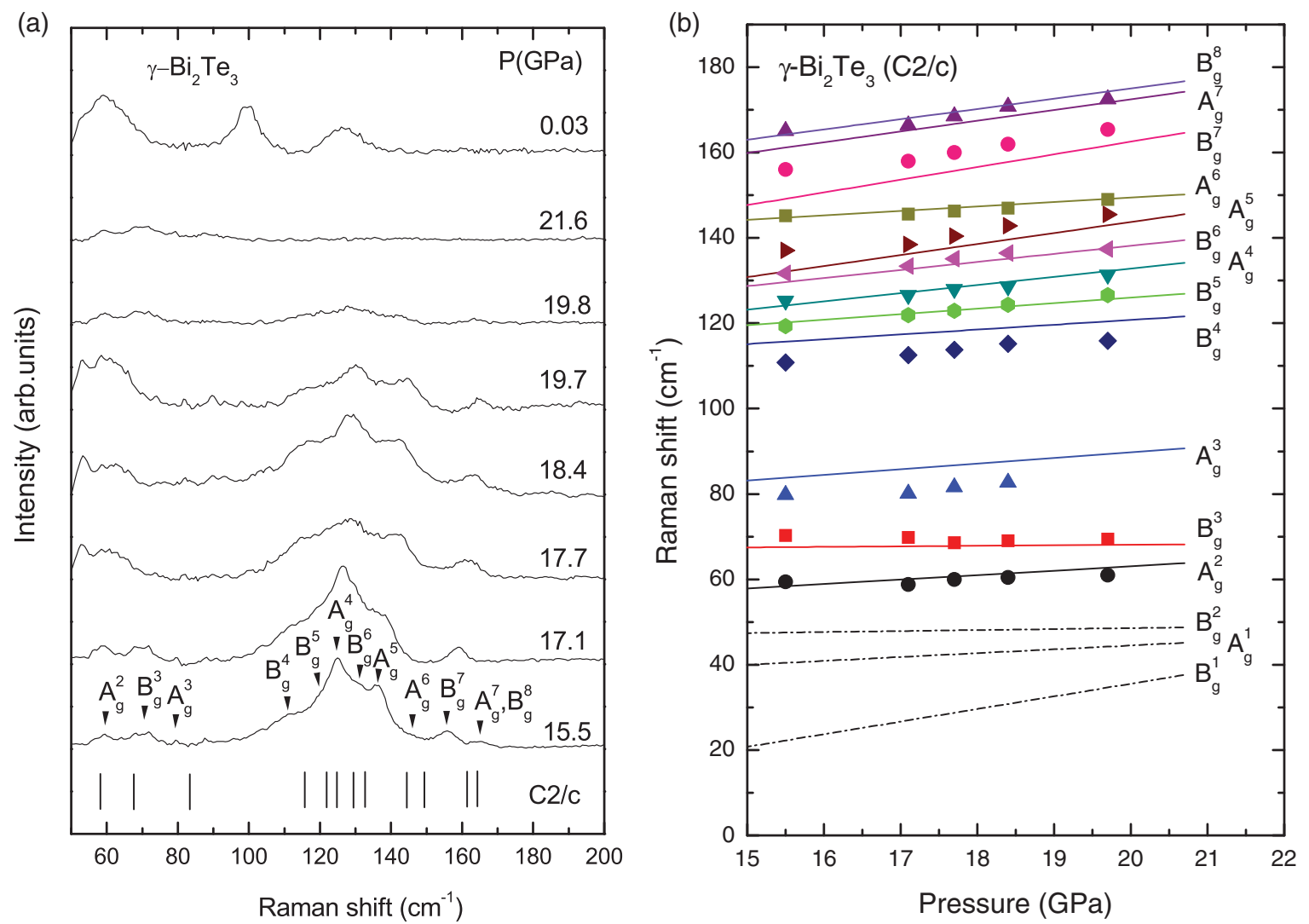

FIG. 8. (Color online) (a) Experimental Raman spectrum of $\gamma-\mathrm{Bi}_{2} \mathrm{Te}_{3}$ at pressures between 15.5 and $21.6 \mathrm{GPa}$ and at ambient pressure after releasing pressure. Bottom marks indicate the calculated frequencies of the Raman-active modes in the $\gamma-\mathrm{Bi}_{2} \mathrm{Te}_{3} \mathrm{phase}$ at $15.5 \mathrm{GPa}$. (b) Experimental pressure dependence of the Raman-mode frequencies in $\gamma-\mathrm{Bi}_{2} \mathrm{Te}_{3}$. Solid (dashed-dotted) curves represent $a b$ initio calculated mode frequencies of modes observed (not observed) in our measurements.

in intensity. Finally, the Raman mode observed around $156 \mathrm{~cm}^{-1}$ has been tentatively attributed to the $B_{\mathrm{g}}^{7}$ mode, and the band at the highest frequency $\left(164 \mathrm{~cm}^{-1}\right)$ at $15.5 \mathrm{GPa}$ has been tentatively attributed to a mixture of the $A_{\mathrm{g}}^{7}$ and $B_{\mathrm{g}}^{8}$ modes since both modes show similar calculated frequencies and pressure coefficients.

As already commented, we have detected a lack of Ramanscattering signal above $20 \mathrm{GPa}$ thus suggesting a phase transition to a Raman-inactive phase above that pressure. The lack of Raman scattering indicates that $\gamma-\mathrm{Bi}_{2} \mathrm{Te}_{3}$ undergoes a complete phase transition toward $\delta-\mathrm{Bi}_{2} \mathrm{Te}_{3}$, and it suggests that the nature of the new phase could be the disordered bcc structure (Im-3m, S.G. 229) recently found in $\mathrm{Bi}_{2} \mathrm{Te}_{3}$ above $14.4 \mathrm{GPa}$ and which dominates the powder x-ray diffraction spectrum above $25 \mathrm{GPa}^{33,34}$ Our Raman measurements suggest that no phase transition to the monoclinic bcc-like $C 2 / m$ phase with nine/ten Bi-coordination occurs. ${ }^{34}$ Note that the main difference between the similar ordered bcc-like $C 2 / m$ and disordered bcc $I m-3 m$ structures is that the bcc-like $C 2 / m$ phase is Raman active with 12 Raman-active modes, while the $I m-3 m$ phase is Raman inactive. Note that on the basis of our Raman measurements alone we cannot assign $\delta-\mathrm{Bi}_{2} \mathrm{Te}_{3}$ to the disordered bcc structure without ambiguity because it is possible that the Raman-scattering signal of the bcc-like $C 2 / \mathrm{m}$ phase is very weak, and we have not been able to measure it. Similar results have been already found in the study of the lattice dynamics of $\mathrm{Sb}_{2} \mathrm{Te}_{3}$ under pressure. ${ }^{51}$

In order to study the structural stability of the different phases of $\mathrm{Bi}_{2} \mathrm{Te}_{3}$ and to support the previous assignments of the different high-pressure phases of $\mathrm{Bi}_{2} \mathrm{Te}_{3}$ we have performed total-energy calculations for $\mathrm{Bi}_{2} \mathrm{Te}_{3}$ with the previously proposed structures. ${ }^{33,34}$ Figure 9(a) and 9(b) show the energy vs volume and the Gibbs free-energy difference at $300 \mathrm{~K}$ vs pressure for the different phases calculated. In Fig. 9(a) it can be observed that the different phases observed in $\mathrm{Bi}_{2} \mathrm{Te}_{3}$ under pressure ${ }^{34}$ cross each other at reduced volumes and therefore are candidates to be observed at different pressures. Therefore, our assignments of the peak frequencies in the preceding paragraphs to the proposed phases are coherent. The only doubt is whether $\delta-\mathrm{Bi}_{2} \mathrm{Te}_{3}$ is the ordered bcc-like $C 2 / m$ nine/ten phase or the disordered bcc $(\mathrm{Im}-3 \mathrm{~m})$ phase. Our total-energy calculations support the phase transition from the $C 2 / c$ phase toward the bcc-like $C 2 / m$ phase. Unfortunately, we have not been able to made calculations for the disordered bcc $I m-3 m$ phase because we cannot easily model disordered phases. Therefore, in order to ensure that these phases are consistent we have plotted in Fig. 9(b) the pressure dependence of the Gibbs free-energy difference at $T=300 \mathrm{~K}$ for the different monoclinic phases with respect to the $R-3 m$, which is taken as reference. The calculated phase-transition pressure for the $R-3 m$ to the $C 2 / m$ phase is $4.5 \mathrm{GPa}$, which compares with the 
TABLE IV. Experimental Raman-mode frequencies and pressure coefficients observed in $\gamma-\mathrm{Bi}_{2} \mathrm{Te}_{3}$ at room temperature at $P_{0}=15.5 \mathrm{GPa}$ as obtained from fits using $\omega(P)=\omega\left(P_{0}\right)+a_{1} \cdot\left(P-P_{0}\right)$. Theoretical (th.) ab initio values for the frequencies and pressure coefficients at $15.5 \mathrm{GPa}$ are also shown for comparison.

\begin{tabular}{|c|c|c|c|c|}
\hline Mode & $\omega\left(P_{0}=15.5 \mathrm{GPa}\right)\left(\mathrm{cm}^{-1}\right)$ & $a_{1}\left(\mathrm{~cm}^{-1} / \mathrm{GPa}\right)$ & $\omega\left(P_{0}=15.5 \mathrm{GPa}\right)($ th. $)\left(\mathrm{cm}^{-1}\right)$ & $a_{1}$ (th.) $\left(\mathrm{cm}^{-1} / \mathrm{GPa}\right)$ \\
\hline $\mathrm{B}_{\mathrm{g}}^{1}$ & & & 22.2 & 2.95 \\
\hline $\mathrm{A}_{\mathrm{g}}^{1}$ & & & 40.4 & 0.90 \\
\hline $\mathrm{B}_{\mathrm{g}}^{2}$ & & & 47.5 & 0.23 \\
\hline $\mathrm{A}_{\mathrm{g}}^{2}$ & 59.0 & 0.45 & 58.4 & 1.04 \\
\hline $\mathrm{B}_{\mathrm{g}}^{3}$ & 70.0 & -0.26 & 67.5 & 0.12 \\
\hline $\mathrm{A}_{\mathrm{g}}^{3}$ & 79.4 & 0.98 & 83.8 & 1.32 \\
\hline $\mathrm{B}_{\mathrm{g}}^{4}$ & 110.8 & 1.29 & 115.7 & 1.13 \\
\hline $\mathrm{B}_{\mathrm{g}}^{5}$ & 119.3 & 1.39 & 120.2 & 1.29 \\
\hline $\mathrm{A}_{\mathrm{g}}^{4}$ & 124.8 & 1.44 & 124.2 & 1.92 \\
\hline $\mathrm{B}_{\mathrm{g}}^{6}$ & 131.7 & 1.44 & 129.7 & 1.89 \\
\hline $\mathrm{A}_{\mathrm{g}}^{5}$ & 137.3 & 2.11 & 132.1 & 2.58 \\
\hline $\mathrm{A}_{\mathrm{g}}^{6}$ & 145.0 & 1.33 & 144.7 & 1.05 \\
\hline $\mathrm{B}_{\mathrm{g}}^{7}$ & 156.3 & 2.29 & 149.2 & 2.97 \\
\hline $\mathrm{A}_{\mathrm{g}}^{7}$ & 164.5 & 1.90 & 161.2 & 2.51 \\
\hline $\mathrm{B}_{\mathrm{g}}^{8}$ & 164.5 & 1.90 & 164.2 & 2.40 \\
\hline
\end{tabular}

experimental value $(7.5 \mathrm{GPa})$. The calculated phase-transition pressure for the $C 2 / m$ to the $C 2 / c$ phase is around $9 \mathrm{GPa}$ while the experimental value is around (15 GPa). In regard to the last phase transition, our calculations show that the monoclinic bcc-like $C 2 / m$ nine-ten structure crosses below the $C 2 / c$ structure near $14 \mathrm{GPa}$. This means that in principle a phase transition from the $C 2 / c$ to the monoclinic bcc-like
$C 2 / m$ phase could be observed at $T=300 \mathrm{~K}$. However, we have calculated the phonons of this bcc-like phase at several pressures between 15 and $18 \mathrm{GPa}$ and have found that there are phonons with negative frequencies, which suggest that this bcc-like phase is not really stable at high pressures. Therefore, we conclude that it is most likely that the $\delta-\mathrm{Sb}_{2} \mathrm{Te}_{3}$ phase is a disordered bcc phase with $I m-3 m$ structure as already found (a)

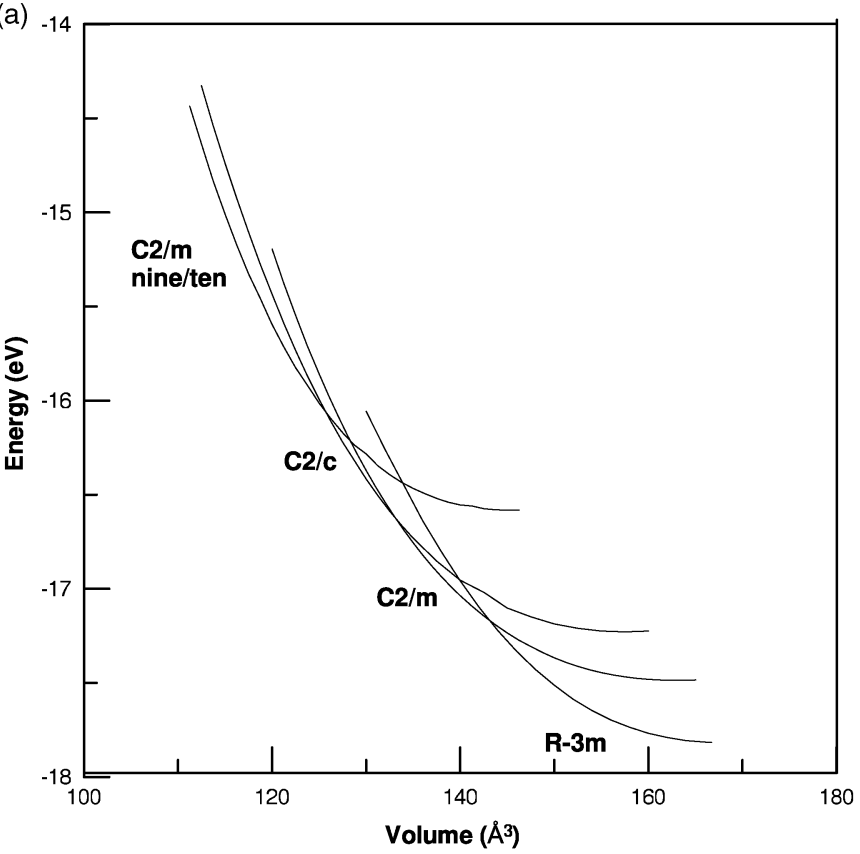

(b)

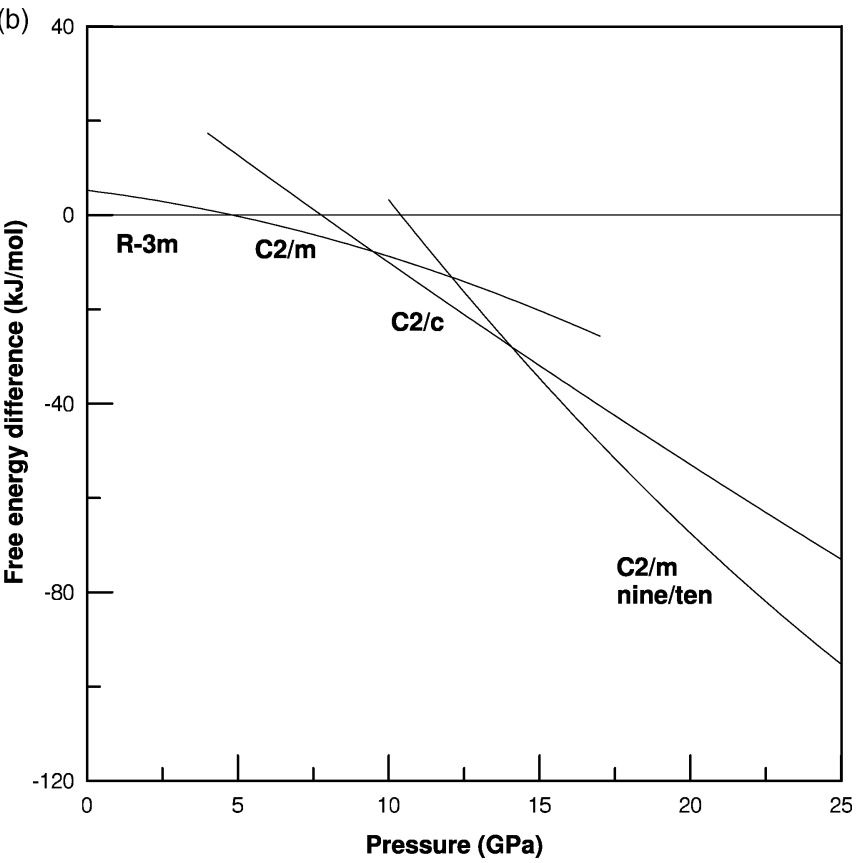

FIG. 9. Theoretical calculation of energy vs volume (a) and and Gibbs free-energy difference at $300 \mathrm{~K}$ as a function of pressure (b) for the $R-3 m, C 2 / m, C 2 / c$, and bcc-like $C 2 / m$ phases of $\mathrm{Bi}_{2} \mathrm{Te}_{3}$. Free energy of $R-3 m$ phase is taken as reference in (b). 
in $\mathrm{Bi}_{2} \mathrm{Te}_{3}$ since it is expected that the Gibbs free energy of the disordered bcc structure is even lower than that of the bcc-like $C 2 / m$ phase. Finally, we must comment that it is possible that the phase transition from the $C 2 / c$ to the $I m-3 m$ phase begins at much lower pressure than $20 \mathrm{GPa}$, as it is observed by powder x-ray diffraction, ${ }^{34}$ but Raman scattering cannot detect it because of the lack of Raman scattering of the latter phase.

\section{CONCLUSIONS}

We have performed room-temperature Raman scattering and optical-absorption measurements and $a b$ initio totalenergy and lattice dynamics calculations in $\mathrm{Bi}_{2} \mathrm{Te}_{3}$ at high pressures. We have shown that $\mathrm{Bi}_{2} \mathrm{Te}_{3}$ exhibits a decrease of the indirect bandgap with a pressure coefficient of $-6 \mathrm{meV} / \mathrm{GPa}$ and that it remains transparent even at 5.5 GPa. We have also found that it follows the sequence of pressure-induced phase transitions up to $23 \mathrm{GPa}$ recently predicted and verified by means of powder $\mathrm{x}$-ray diffraction measurements ${ }^{33,34}$ and that were also confirmed to occur in $\mathrm{Sb}_{2} \mathrm{Te}_{3}$ under pressure. ${ }^{51}$ We have also found that a less positive pressure coefficient is observed in $\alpha$ $\mathrm{Bi}_{2} \mathrm{Te}_{3}$ around 4.0 GPa due to an electronic-topology transition in the rhombohedral phase, as already evidenced in other type of measurements $20,29,31,32,35,36$ and in Raman-scattering measurements in $\mathrm{Sb}_{2} \mathrm{Te}_{3}$ under pressure. ${ }^{51}$ The pressure dependence of the experimental and calculated Raman-mode frequencies in the different phases are reported and found to be in good agreement with each other and only the low-frequency modes of each phase were not observed likely because of their weak intensity. Finally, we must note that on fully releasing the pressure the sample returns to the rhombohedral structure below $5 \mathrm{GPa}$ as previously reported. ${ }^{34}$ We hope the present work will further stimulate new measurements in $\mathrm{Bi}_{2} \mathrm{Te}_{3}$ under high pressure. In general more work on layered chalcogenide compounds is needed to understand the mechanism of their pressure-induced electronic-topological phase transitions and the subtle effects on their structures and their properties.

\section{ACKNOWLEDGMENTS}

This work has been done under financial support from Spanish MICINN under projects MAT2008-06873-C0202, MAT2007-66129, Prometeo/2011-035, MAT2010-21270C04-03/04, and CSD2007-00045 and supported by the Ministry of Education, Youth and Sports of the Czech Republic (MSM 0021627501). E P-G acknowledges the financial support of the Spanish MEC under a FPI fellowship. Supercomputer time has been provided by the Red Española de Supercomputación (RES) and the MALTA cluster. *rovilap@ fis.upv.es

${ }^{1}$ G. J. Snyder and E. S. Tobere, Nat. Mater. 7, 105 (2008).

${ }^{2}$ D. M. Rowe, CRC Handbook of Thermoelectrics, (CRC Press Inc., New York, 1995).

${ }^{3}$ R. Venkatasubramanian, E. Siivola, T. Colpitts, and B. O'Quinn, Nature 413, 597 (2001)

${ }^{4}$ M. Dresselhaus, G. Dresselhaus, X. Sun, Z. Zhang, S. Cronin, and T. Koga, Phys. Solid State 41, 679 (1999).

${ }^{5}$ D. Teweldebrhan, V. Goyal, M. Rahman, and A. A. Balandin, Appl. Phys. Lett. 96, 053107 (2010).

${ }^{6}$ A. Adam, Mat. Res. Bull. 42, 1986 (2007).

${ }^{7}$ T. J. Scheidemantel, J. F. Meng, and J. V. Badding, J. Phys. Chem. Solids 66, 1744 (2005).

${ }^{8}$ H. Zhang, C. X. Liu, X. L. Qi, X. Dai, Z. Fang, and S. C. Zhang, Nature Phys. 5, 438 (2009).

${ }^{9}$ M. Z. Hassan and C. L. Kane, Rev. Mod. Phys. 82, 3045 (2010).

${ }^{10}$ J. E. Moore, Nature 464, 194 (2010).

${ }^{11}$ Y. Xia, D. Qian, D. Hsieh, L. Wray, A. Pal, H. Lin, A. Bansil, D. Grauer, Y. S. Hor, R. J. Cava, and M. Z. Hassan, Nat. Phys. 5, 398 (2009).

${ }^{12}$ H. Zhang, C. X. Liu, X. L. Qi, X. Dai, Z. Fang, and S. C. Zhang, Nat. Phys. 5, 438 (2009).

${ }^{13}$ Y. L. Chen, J. G. Analytis, J. H. Chu, Z. K. Liu, S. K. Mo, X. L. Qi, H. J. Zhang, D. H. Lu, X. Dai, Z. Fang, S. C. Zhang, I. R. Fisher, Z. Hussain, and Z. X. Shen, Science 325, 178 (2009).

${ }^{14}$ J. V. Badding, J. F. Meng, and D. A. Polvani, Chem. Mater. 10, 2889 (1998).

${ }^{15}$ D. A. Polvani, J. F. Meng, N. V. Chandra Shekar, J. Sharp, and J. V. Badding, Chem. Mater. 13, 2068 (2001).

${ }^{16}$ N. V. Chandra Shekar, D. A. Polvani, J. F. Meng, and J. V. Badding, Physica B 358, 14 (2005).
${ }^{17}$ S. V. Ovsyannikov, V. V. Shchennikov, G. V. Vorontsov, A. Y. Manakov, A.Y Likhacheva, and V. A. Kulbachinskii, J. Appl. Phys. 104, 053713 (2008).

${ }^{18}$ S. V. Ovsyyannikov and V. V. Shchennikov, Chem. Mater. 22, 635 (2010).

${ }^{19}$ C.-Y. Li, A. L. Ruoff, and C. W. Spencer, J. Appl. Phys. 32, 1733 (1961).

${ }^{20}$ E. S. Itskevich, S. V. Popova, and E. Ya. Atabaeva, Dokl. Akad. Nauk SSSR 153, 306 (1963).

${ }^{21}$ L. F. Vereshchagin, E. Ya. Atabaeva, and N. A. Bedeliani, Sov. Phys. Solid State 13, 2051 (1972).

${ }^{22}$ L. G. Khvostantsev, A. I Orlov, N. Kh. Abrikosov, and L. D. Ivanova, Phys. Stat. Solidi a 58, 37 (1980).

${ }^{23}$ N. Sakai, T. Kajiwara, K. Takemura, S. Minomura, and Y. Fujii, Solid State Commun. 40, 1045 (1981).

${ }^{24}$ L. G. Khvostantsev, A. I Orlov, N. Kh. Abrikosov, and L. D. Ivanova, Phys. Stat. Solidi a 89, 301 (1985).

${ }^{25} \mathrm{M}$. Bartkowiak and G. D. Mahan, $18^{\text {th }}$ International Conference on Thermoelectrics, IEEE 713 (1999).

${ }^{26}$ T. Thonhauser, T. J. Scheidemantel, J. O. Sofo, J. V. Badding, and G. D. Mahan, Phys. Rev. B 68, 085201 (2003).

${ }^{27}$ T. Thonhauser, Solid State Commun. 129, 249 (2004).

${ }^{28}$ M. Einaga, Y. Tanabe, A. Nakayama, A. Ohmura, F. Ishikawa, and Yuh Yamada, J. Phys.: Conf. Ser. 215, 012036 (2010).

${ }^{29}$ J. L. Zhang, S. J. Zhang, H. M. Weng, W. Zhang, L. X. Yang, Q. Q. Liu, S. M. Feng, X. C. Wang, R. C. Yu, L. Z. Cao, L. Wang, W. G. Yang, H. Z. Liu, W. Y. Zhao, S. C. Zhang, X. Dai, Z. Fang, and C. Q. Jin, Proc. Nat. Acad. Sci. 108, 24 (2011).

${ }^{30}$ C. Zhang, L. Sun, Z. Chen, X. Zhou, Q. Wu, W. Yi, J. Guo, X. Dong, and Z. Zhao, Phys. Rev. B 83, 140504 (2011). 
${ }^{31}$ M. K. Jacobsen, R. S. Kumar, A. L. Cornelius, S. V. Sinogeiken, and M. F. Nicol, AIP Conf. Proc. 955, 171 (2007).

${ }^{32}$ A. Nakayama, M. Einaga, Y. Tanabe, S. Nakano, F. Ishikawa, and Y. Yamada, High. Press. Res. 29, 245 (2009).

${ }^{33}$ M. Einaga, F. Ishikawa, A. Ohmura, A. Nakayama, Y. Yamada, and S. Nakano, Phys. Rev. B 83, 092102 (2011).

${ }^{34}$ L. Zhu, H. Wang, Y. C. Wang, J. Lv, Yanmei Ma, Q. L. Cui, Yanming Ma, and G. T. Zou, Phys. Rev. Lett. 106, 145501 (2011).

${ }^{35}$ E. S. Itskevich, L. M. Kashirskaya, and V. F. Kraidenov, Semicond. 31, 276 (1997).

${ }^{36}$ A. Polian, M. Gauthier, S. M. Souza, D. M. Trichês, J. Cardoso de Lima, and T. A. Grandi, Phys. Rev. B 83, 113106 (2011).

${ }^{37}$ I. M. Lifshitz, Sov. Phys. JETP 11, 1130 (1960).

${ }^{38}$ L. Dagens, J. Phys. F: Met. Phys. 8, 4496 (1978).

${ }^{39}$ L. Dagens and C. Lopez-Rios, J. Phys. F: Met. Phys. 9, 2195 (1979).

${ }^{40}$ A. F. Goncharov, V. V. Struzhkin, Physica B 385, 117 (2003).

${ }^{41}$ D. Antonangeli, D. L. Farber, A. H. Said, L. R. Benedetti, C. M. Aracne, A. Landa, P. Söderlind, and J. E. Klepeis, Phys. Rev. B 82, 132101 (2010).

${ }^{42}$ W. Richter, H. Köhler, and C. R. Becker, Phys. Stat. Solidi B 84, 619 (1977).

${ }^{43}$ H. Rauh, R. Geick, H. Köhler, N. Nücker, and N. Lehner, J. Phys. C: Solid State Phys. 14, 2705 (1981).

${ }^{44}$ W. Kullmann, G. Eichhorn, H. Rauh, R. Geick, G. Eckold, and U. Steigenberger, Phys. Status Solidi B 162, 125 (1990).

${ }^{45}$ K. M. F. Shahil, M. Z. Hossain, D. Teweldebrhan, and A. A. Balandin, Appl. Phys. Lett. 96, 153103 (2010).

${ }^{46}$ J. O. Jenkins, J. A. Rayne, and R. W. Ure Jr., Phys. Rev. B 5, 3171 (1972).

${ }^{47}$ B. L. Huang and M. Kaviany, Phys. Rev. B 77, 125209 (2008).

${ }^{48}$ B. Qiu and X. Ruan, Phys. Rev. B 80, 165203 (2009).

${ }^{49}$ W. Cheng and S. F. Ren, Phys. Rev. B 83, 094301 (2011).

${ }^{50}$ W. Kullmann, J. Geurts, W. Richter, N. Lehner, H. Rauh, U. Steigenberger, G. Eichhorn, and R. Geick, Phys. Status Solidi B 125, 131 (1984).

${ }^{51}$ O. Gomis, R. Vilaplana, F. J. Manjón, P. Rodríguez-Hernández, E. Pérez-González, A. Muñoz, V. Kucek, and C. Drasar (to be published).

${ }^{52}$ J. Bludska, I. Jakubec, C. Drasar, P. Lostak, and J. Horak, Philos. Mag. 87, 325 (2007).

${ }^{53}$ G. J. Piermarini, S. Block, and J. D. Barnett, J. Appl. Phys. 44, 5377 (1973).

${ }^{54}$ D. Errandonea, Y. Meng, M. Somayazulu, and D. Hausermann, Physica B 355, 116 (2005).

${ }^{55}$ K. Syassen, High Press. Res. 28, 75 (2008).
${ }^{56}$ P. Hohenberg and W. Kohn, Phys. Rev. 136, 3864 (1964).

${ }^{57}$ G. Kresse and J. Hafner, Phys. Rev. B 47, 558 (1993); G. Kresse and J. Hafner, Phys. Rev. B 49, 14251 (1994); G. Kresse and J. Furthmüller, Comput. Mat. Sci. 6, 15 (1996); G. Kresse and J. Furthmüller, Phys. Rev. B 54, 11169 (1996).

${ }^{58}$ P. E. Blöchl, Phys. Rev. B 50, 17953 (1994); G. Kresse and D. Joubert, Phys. Rev. B 59, 1758 (1999).

${ }^{59}$ J. P. Perdew, A. Ruzsinszky, G. I. Csonka, O. A. Vydrow, G. E. Scuseria, L. A. Constantin, X. Zhou, and K. Burke, Phys. Rev. Lett. 100, 136406 (2008).

${ }^{60}$ A. Mujica, A. Rubio, A. Muñoz, and R. J. Needs, Rev. Mod. Phys. 79, 863 (2003).

${ }^{61}$ M. A. Blanco, E. Francisco, and V. Luaña, Comp. Phys. Commun. 158, 57 (2004).

${ }^{62} \mathrm{~K}$. Parlinski, computer code PHONON [http://wolf.ifj.edu.pl/ phonon].

${ }^{63}$ See Supplemental Material at http://link.aps.org/supplemental/ 10.1103/PhysRevB.84.104112 for calculation details regarding IRactive modes.

${ }^{64}$ J. Black, E. M. Comwell, L. Seigle, and C. W. Spencer, J. Phys. Chem. Solids 2, 240 (1957).

${ }^{65}$ I. G. Austin, Proc. Phys. Rev. 72, 545 (1958).

${ }^{66}$ D. L. Greenaway and G. Harbeke, J. Phys. Chem. Solids 26, 1585 (1965).

${ }^{67}$ M. Kim, A. J. Freeman and C. B. Geller, Phys. Rev. B 72, 035205 (2005).

${ }^{68}$ H. Kohler, Phys. Status Solidi B 73, 95 (1976).

${ }^{69}$ E. S. Itskevich, L. M. Kashirskaya, and V. F. Kraidenov, Semiconductors 31, 276 (1997).

${ }^{70}$ G. Herzberg, Molecular Spectra and Molecular Structure II: InfraRed and Raman Spectra, (D. Van Nostrand Co. Inc., New York, 1945).

${ }^{71}$ V. Russo, A. Bailini, M. Zamboni, M. Passoni, C. Conti, C. S. Casari, A. Li Bassi, and C. E. Bottani, J. Raman Spectrosc. 39, 205 (2008).

${ }^{72}$ L. M. Gonsalves, C. Couto, P. Alpuim, A. G. Rolo, F. Völkein, and J. H. Correia, Thin Solid Films 518, 2816 (2010).

${ }^{73}$ Y. Liang, W. Wang, B. Zeng, G. Zhang, J. Huang, J. Li, T. Li, Y. Song, and X. Zhang, J. Alloys Comp. 509, 5147 (2011).

${ }^{74}$ C. Ulrich, M. A. Mroginski, A. R. Goñi, A. Cantarero, U. Schwarz, V. Muñoz, and K. Syassen, Phys. Stat. Sol. 198, 121 (1996).

${ }^{75}$ A. M. Kubelikov, H. P. Olijnyk, A. P. Jephcoat, Z. Y. Salaeva, S. Onari, and K. R. Allakverdiev, Phys. Stat. Sol (B) 235, 517 (2003). 